\title{
AVERAGING PRINCIPLE FOR ONE DIMENSIONAL STOCHASTIC BURGERS EQUATION
}

\author{
ZHAO DONG ${ }^{1,2}$, XIAOBIN SUN ${ }^{3}$, HUI XIAO ${ }^{1,2,4}$, AND JIANLIANG ZHAI $^{5}$
}

\begin{abstract}
In this paper, we consider the averaging principle for one dimensional stochastic Burgers equation with slow and fast time-scales. Under some suitable conditions, we show that the slow component strongly converges to the solution of the corresponding averaged equation. Meanwhile, when there is no noise in the slow component equation, we also prove that the slow component weakly converges to the solution of the corresponding averaged equation with the order of convergence $1-r$, for any $0<r<1$.
\end{abstract}

\section{INTRODUCTION}

Many multiscale problems arise from material sciences, chemistry, fluids dynamics, biology, ecology, climate dynamics and other application areas, see, e.g., [1, 12, 19, 23, 27, 29, 36] and references therein. E and Engquist [12] pointed out "Problems in these areas are often multiphysics in nature; namely, the processes at different scales are governed by physical laws of different character: for example, quantum mechanics at one scale and classical mechanics at another." For instance, dynamics of chemical reaction networks often take place on notably different times scales, from the order of nanoseconds $\left(10^{-9} \mathrm{~s}\right)$ to the order of several days, the use of two-time or multi-time scales is common. Another example with multiple time scales is that of protein folding. While the time scale for the vibration of the covalent bonds is on the order of femtoseconds $\left(10^{-15} \mathrm{~s}\right)$, folding time for the proteins may very well be on the order of seconds.

Many two-time scale/slow-fast systems can be formally written as

$$
\left\{\begin{array}{l}
d X_{t}^{\varepsilon}=b_{1}\left(X_{t}^{\varepsilon}, Y_{t}^{\varepsilon}\right) d t+\sigma_{1}\left(X_{t}^{\varepsilon}, Y_{t}^{\varepsilon}\right) d W_{t}^{1}, \quad X_{0}^{\varepsilon}=x \\
d Y_{t}^{\varepsilon}=\frac{1}{\varepsilon} b_{2}\left(X_{t}^{\varepsilon}, Y_{t}^{\varepsilon}\right) d t+\frac{1}{\sqrt{\varepsilon}} \sigma_{2}\left(X_{t}^{\varepsilon}, Y_{t}^{\varepsilon}\right) d W_{t}^{2}, \quad Y_{0}^{\varepsilon}=y,
\end{array}\right.
$$

where $W_{t}^{1}, W_{t}^{2}$ are independent Wiener processes, and the small parameter $\varepsilon$ quantifies the ratio of the $X^{\varepsilon}$ and $Y^{\varepsilon}$ time scales. For many practical problems, it is of interest to study the behavior of the system (1.1) for $\varepsilon<<1$, and how dynamics of this system depends on $\varepsilon$ as $\varepsilon \rightarrow 0$. However, since $\varepsilon<<1$, it is often very difficult to directly calculate $X^{\varepsilon}$, and systems of this type are problematic for computer simulations. The averaging principle can be applied to solve these problems of this type. Roughly speaking, if the dynamics for $Y^{\varepsilon}$

\footnotetext{
${ }^{1}$ RCSDS, Academy of Mathematics and Systems Science, Chinese Academy of Sciences, Beijing 100190, China

${ }^{2}$ School of Mathematical Sciences, University of Chinese Academy of Sciences, Beijing 100049, China

${ }^{3}$ School of Mathematics and Statistics, Jiangsu Normal University, Xuzhou, 221116, China

${ }^{4}$ Université de Bretagne-Sud, LMBA UMR CNRS 6205, Vannes, France

${ }^{5}$ School of Mathematical Science, University of Science and Technology of China, Hefei, 230026, China

2000 Mathematics Subject Classification. Primary 34D08, 34D25; Secondary 60H20.

Key words and phrases. Stochastic Burgers' equation; Averaging principle; Ergodicity; Invariant measure; Strong convergence; Weak convergence.
} 
with $X^{\varepsilon}=x$ fixed has an invariant probability measure $\mu^{x}(d y)$ and the following integrals exist

$$
\bar{b}_{1}(x):=\int b_{1}(x, y) \mu^{x}(d y), \quad \bar{\sigma}_{1}(x):=\int \sigma_{1}(x, y) \mu^{x}(d y)
$$

then under appropriate assumptions on all coefficients in the system (1.1), the effective dynamics for $X^{\varepsilon}$ in the limit of $\varepsilon \rightarrow 0$ is a stochastic differential equation:

$$
d \bar{X}_{t}=\bar{b}_{1}\left(\bar{X}_{t}\right) d t+\bar{\sigma}_{1}\left(\bar{X}_{t}\right) d W_{t}^{1}, \quad \bar{X}_{0}=x .
$$

The theory of averaging principle has a long history and rich results. Bogoliubov and Mitropolsky [2] first studied the averaging principle for the deterministic systems. Later on, the theory of averaging principle for stochastic differential equations was firstly established by Khasminskii [21]. Since then, averaging principle for stochastic reaction-diffusion systems has become an active research area which attracted much attention. For example, Cerrai and Freidlin [5] proved the averaging principle for a general class of stochastic reactiondiffusion systems, which extended the classical Khasminskii-type averaging principle for finite dimensional systems to infinite dimensional systems. Recently, based on the averaging principle, the fast flow asymptotics for a stochastic reaction-diffusion-advection equation are obtained by Cerrai and Freidlin [6]. We refer to [3, 4, 13, 15, 16, 17, 18, 22, 24, 33, 34, 35] and references therein for more interesting results on this topic.

To the best of our knowledge, there are rarely studies to deal with highly nonlinear term on this topic. In this paper, we are interested in studying the averaging principle for one dimensional stochastic Burgers, i.e., considering the following stochastic slow-fast system on the interval $[0,1]$ :

$$
\left\{\begin{array}{l}
\frac{\partial X_{t}^{\varepsilon}(\xi)}{\partial t}=\left[\Delta X_{t}^{\varepsilon}(\xi)+\frac{1}{2} \frac{\partial}{\partial \xi}\left(X_{t}^{\varepsilon}(\xi)\right)^{2}+f\left(X_{t}^{\varepsilon}(\xi), Y_{t}^{\varepsilon}(\xi)\right)\right]+\frac{\partial W^{Q_{1}}}{\partial t}(t, \xi), \quad X_{0}^{\varepsilon}=x \\
\frac{\partial Y_{t}^{\varepsilon}(\xi)}{\partial t}=\frac{1}{\varepsilon}\left[\Delta Y_{t}^{\varepsilon}(\xi)+g\left(X_{t}^{\varepsilon}(\xi), Y_{t}^{\varepsilon}(\xi)\right)\right]+\frac{1}{\sqrt{\varepsilon}} \frac{\partial W^{Q_{2}}}{\partial t}(t, \xi), \quad Y_{0}^{\varepsilon}=y \\
X_{t}^{\varepsilon}(0)=X_{t}^{\varepsilon}(1)=Y_{t}^{\varepsilon}(0)=Y_{t}^{\varepsilon}(1)=0
\end{array}\right.
$$

where $\varepsilon>0$ is a small parameter describing the ratio of time scales between the slow component $X_{t}^{\varepsilon}$ and fast component $Y_{t}^{\varepsilon}$. The coefficients $f$ and $g$ satisfy some suitable conditions. $\left\{W_{t}^{Q_{1}}\right\}_{t \geqslant 0}$ and $\left\{W_{t}^{Q_{2}}\right\}_{t \geqslant 0}$ are $L^{2}(0,1)$-valued mutually independent $Q_{1}$ and $Q_{2^{-}}$ Wiener processes.

For any $t \in[0, T]$, as $\varepsilon \rightarrow 0$, the slow component $X_{t}^{\varepsilon}$ in (1.2) converges to $\bar{X}_{t}$, which is the solution of the averaged equation:

$$
\left\{\begin{array}{l}
d \bar{X}_{t}=\Delta \bar{X}_{t} d t+\frac{1}{2} \frac{\partial}{\partial \xi}\left(\bar{X}_{t}\right)^{2} d t+\bar{f}\left(\bar{X}_{t}\right) d t+d W^{Q_{1}}(t) \\
\bar{X}_{0}=x
\end{array}\right.
$$

with the average

$$
\bar{f}(x)=\int_{L^{2}(0,1)} f(x, y) \mu^{x}(d y)
$$

where $\mu^{x}$ denotes the unique invariant measure for the fast component equation with frozen slow component variable $x$ (see the equation (3.25) for details).

We aim to study the rate of convergences of the process $X^{\varepsilon}$ to $\bar{X}$, both in the strong convergence sense and in the weak convergence sense. Under some appropriate conditions, the result of strong convergence is stated as follows: 
- For any $x \in H^{\alpha}(0,1)$ with $\alpha \in\left(1, \frac{3}{2}\right]$ and $y \in L^{2}(0,1), p>0, T>0$, there exists a positive constant $C$ which is independent of $\varepsilon$ such that

$$
\mathbb{E}\left(\sup _{0 \leqslant t \leqslant T}\left\|X_{t}^{\varepsilon}-\bar{X}_{t}\right\|^{2 p}\right) \leqslant C\left(\frac{1}{-\log \varepsilon}\right)^{\frac{1}{4 p}} \rightarrow 0, \quad \text { as } \varepsilon \rightarrow 0 .
$$

Here we denote by $\|\cdot\|$ the norm of $L^{2}(0,1)$.

If $Q_{1}=0$ in the system (1.2), then under some conditions, the result of weak convergence is stated as follows:

- For any $x \in H^{\theta}(0,1)$ with $\theta \in(0,1], y \in L^{2}(0,1), \phi \in C_{b}^{2}\left(L^{2}(0,1)\right), r \in(0,1)$, $\delta \in\left(0, \frac{1}{2}\right), t \in[0, T]$, there exists a positive constant $C$ which is independent of $\varepsilon$ such that

$$
\left|\mathbb{E} \phi\left(X_{t}^{\varepsilon}\right)-\mathbb{E} \phi\left(\bar{X}_{t}\right)\right| \leqslant C\left(1+t^{-\theta+\frac{\theta^{2}}{1+\delta}}\right) \varepsilon^{1-r} .
$$

Comparing with the strong convergence, while the requirement on the regularity of initial value $x$ in weak convergence is weaker, the rate of the convergence is pleasant in this case. The idea of the proof follows a procedure inspired by [3], in which the authors considered a relative simple framework (without the nonlinear term and with $f$ being bounded ). In our case, to deal with the nonlinear term and unbounded $f$ is a nontrivial task.

The proof of (1.4) is based on the Khasminskii argument introduced in [21], but it is clearly more involved than in [21], as it concerns the nonlinear term in the Burgers' equation and unbounded $f$. To be precise, we split the interval $[0, T]$ into some subintervals of size $\delta>0$, then on each interval $[k \delta,(k+1) \delta)), k \geqslant 0$, we construct an auxiliary process $\left(\hat{X}_{t}^{\varepsilon}, \hat{Y}_{t}^{\varepsilon}\right)$, $t \in[k \delta,(k+1) \delta)$ ), associated with the system (1.2). Based on the exponential ergodicity of the fast component equation with frozen slow component $x$ in the system (1.2), by controlling the error between $\hat{X}_{t}^{\varepsilon}$ and $X_{t}^{\varepsilon}$, allows us to deduce (1.4). The biggest challenge in studying the strong convergence (1.4) is to deal with the nonlinear term. To overcome this difficulty, we first give some estimates of the slow component $X_{t}^{\varepsilon}$ and fast component $Y_{t}^{\varepsilon}$ in $L^{2}(0,1)$. Secondly, by using the smoothness of semigroup $e^{t \Delta}$ and the interpolation inequality, we can further obtain $\sup _{\varepsilon \in(0,1)} \mathbb{E} \sup _{t \in[0, T]}\left|X_{t}^{\varepsilon}\right|_{\alpha}^{p} \leqslant C_{p, T}$, which is a key step for proving (1.4), where $|\cdot|_{\alpha}$ is the Sobolev norm. Finally, we obtain the result by applying the skill of stopping time and following the procedure inspired by [15].

To obtain the weak convergence (1.5), we use the asymptotic expansion with respect to $\varepsilon$ of the solution to the Kolmogorov equation corresponding to the system (1.2). However, some problems appear since the operator $\Delta$ is unbounded in the Kolmogorov equation. To overcome this difficulty, following the approach used in [3], we first use the Galerkin approximation to reduce the infinite dimensional problem to a finite dimensional one, then the remaining part is to establish the rate of convergence with some bounds which is independent of the dimension. Note that instead of using the asymptotic expansion of the solution to the Kolmogorov equation, an alternative martingale approach was applied to prove the weak convergence for stochastic reaction-diffusion equations with unbounded multiplicative noise $[4]$.

Finally, we refer that, in recent years, there are many interesting results for stochastic Burger's equation $[7,8,9,10,11,14,20,25,26,30,31,32]$.

The rest of the paper is organized as follows. In Section 2, under some suitable assumptions, we formulate our main results. Section 3 and Section 4 are devoted to proving the 
strong convergence and weak convergence, respectively. In the Appendix 5, we recall some useful inequalities.

Throughout the paper, $C, C_{p}$ and $C_{p, T}$ will denote positive constants which may change from line to line, where $C_{p}$ depends on $p, C_{p, T}$ depends on $p, T$.

\section{Notations And MAin RESUlts}

Let $L^{2}:=L^{2}(0,1)$ be the space of square integrable real-valued functions on the interval $[0,1]$. The norm and the inner product on $L^{2}$ are denoted by $\|\cdot\|$ and $\langle\cdot, \cdot\rangle$, respectively. The space $C_{b}^{2}\left(L^{2}\right)$ is the functions from $L^{2}$ to $\mathbb{R}$, which are twice continuously differentiable with first and second bounded derivative. For $k \in \mathbb{N}, W^{k, 2}(0,1)$ is the Sobolev space of all functions in $L^{2}$ whose differentials belong to $L^{2}$ up to the order $k$. The usual Sobolev space $W^{k, 2}(0,1)$ can be extended to the $W^{s, 2}(0,1)$, for $s \in \mathbb{R}$. Set $H^{k} \hat{=} W^{k, 2}(0,1)$ and denote by $H_{0}^{1}$ the subspace of $H^{1}$ of all functions whose trace at 0 and 1 vanishes. The Laplacian operator $\Delta$ is given by

$$
A x=\Delta x=\frac{\partial^{2}}{\partial \xi^{2}} x, \quad x \in \mathscr{D}(A)=H^{2} \cap H_{0}^{1} .
$$

It is well known that $\Delta$ is the infinitesimal generator of a strongly continuous semigroup $\left\{e^{t \Delta}\right\}_{t \geqslant 0}$. The eigenfunctions of $\Delta$ are given by $e_{k}(\xi)=\sqrt{2} \sin (k \pi \xi), \xi \in[0,1], k \in \mathbb{N}$, with the corresponding eigenvalues $\lambda_{k}=-k^{2} \pi^{2}$. The operator $\Delta$ satisfies the smoothing property: for any $s_{1}, s_{2} \in \mathbb{R}$ with $s_{1} \leqslant s_{2}$,

$$
\left|e^{t \Delta} z\right|_{H^{s_{2}}} \leqslant C\left(1+t^{\left(s_{1}-s_{2}\right) / 2}\right)|z|_{H^{s_{1}}}, \quad \text { for } z \in H^{s_{1}}
$$

For any $\alpha \in \mathbb{R},(-A)^{\alpha}$ is the power of the operator $-A$, and $|\cdot|_{\alpha}$ is the norm of $\mathscr{D}\left((-A)^{\alpha / 2}\right)$ which is equivalent to the norm of $H^{\alpha}$.

Define the bilinear operator $B(x, y): L^{2} \times H_{0}^{1} \rightarrow H_{0}^{-1}$ by

$$
B(x, y)=x \cdot \partial_{\xi} y,
$$

and the trilinear operator $b(x, y, z): L^{2} \times H_{0}^{1} \times L^{2} \rightarrow \mathbb{R}$ by

$$
b(x, y, z)=\int_{0}^{1} x(\xi) \partial_{\xi} y(\xi) z(\xi) d \xi
$$

For convenience, set $B(x)=B(x, x)$, for $x \in H_{0}^{1}$.

With the above notations, the system (1.2) can be rewritten as:

$$
\left\{\begin{array}{l}
d X_{t}^{\varepsilon}=\left[A X_{t}^{\varepsilon}+B\left(X_{t}^{\varepsilon}\right)+f\left(X_{t}^{\varepsilon}, Y_{t}^{\varepsilon}\right)\right] d t+d W_{t}^{Q_{1}}, \quad X_{0}^{\varepsilon}=x \\
d Y_{t}^{\varepsilon}=\frac{1}{\varepsilon}\left[A Y_{t}^{\varepsilon}+g\left(X_{t}^{\varepsilon}, Y_{t}^{\varepsilon}\right)\right] d t+\frac{1}{\sqrt{\varepsilon}} d W_{t}^{Q_{2}}, \quad Y_{0}^{\varepsilon}=y \\
X_{t}^{\varepsilon}(0)=X_{t}^{\varepsilon}(1)=Y_{t}^{\varepsilon}(0)=Y_{t}^{\varepsilon}(1)=0 .
\end{array}\right.
$$

Here, the $Q_{1}$-Wiener process $W_{t}^{Q_{1}}$ is given by

$$
W_{t}^{Q_{1}}=\sum_{k=1}^{\infty} \sqrt{\alpha_{k}} \beta_{t}^{k} e_{k}, \quad t \geqslant 0
$$

where $\alpha_{k} \geqslant 0$ satisfies $\operatorname{Tr} Q_{1}:=\sum_{k=1}^{\infty} \alpha_{k}<+\infty$, and $\left\{\beta^{k}\right\}_{k \in \mathbb{N}}$ is a sequence of mutually independent standard Brownian motions. Throughout this paper, we assume that $W_{t}^{Q_{2}}$ also has a similar decomposition as in (2.3) with $\operatorname{Tr} Q_{2}<\infty$. Note that $W_{t}^{Q_{1}}$ and $W_{t}^{Q_{2}}$ are independent.

We impose the global Lipschitz condition on the functions $f, g: L^{2} \times L^{2} \rightarrow L^{2}$ in (2.2). 
A1. There exist two constants $L_{f}, L_{g}>0$ such that for any $x_{1}, x_{2}, y_{1}, y_{2} \in L^{2}$,

$$
\begin{gathered}
\left\|f\left(x_{1}, y_{1}\right)-f\left(x_{2}, y_{2}\right)\right\| \leqslant L_{f}\left(\left\|x_{1}-x_{2}\right\|+\left\|y_{1}-y_{2}\right\|\right), \\
\left\|g\left(x_{1}, y_{1}\right)-g\left(x_{2}, y_{2}\right)\right\| \leqslant L_{g}\left(\left\|x_{1}-x_{2}\right\|+\left\|y_{1}-y_{2}\right\|\right) .
\end{gathered}
$$

Following the standard approach developed in [9], one can verify that under the condition A1, there exists a unique mild solution to the system (2.2). More specifically, for any given initial value $x, y \in L^{2}$, and $T>0$, there exist a unique $X^{\varepsilon} \in C\left([0, T] ; L^{2}\right) \cap L^{2}\left(0, T ; H_{0}^{1}\right)$ and a unique $Y^{\varepsilon} \in C\left([0, T] ; L^{2}\right) \cap L^{2}\left(0, T ; H_{0}^{1}\right)$ satisfying

$$
\left\{\begin{array}{l}
X_{t}^{\varepsilon}=e^{t A} x+\int_{0}^{t} e^{(t-s) A} B\left(X_{s}^{\varepsilon}\right) d s+\int_{0}^{t} e^{(t-s) A} f\left(X_{s}^{\varepsilon}, Y_{s}^{\varepsilon}\right) d s+\int_{0}^{t} e^{(t-s) A} d W_{s}^{Q_{1}}, \\
Y_{t}^{\varepsilon}=e^{t A / \varepsilon} y+\frac{1}{\varepsilon} \int_{0}^{t} e^{(t-s) A / \varepsilon} g\left(X_{s}^{\varepsilon}, Y_{s}^{\varepsilon}\right) d s+\frac{1}{\sqrt{\varepsilon}} \int_{0}^{t} e^{(t-s) A / \varepsilon} d W_{s}^{Q_{2}} .
\end{array}\right.
$$

We need the following dissipative condition, which allows us to obtain the exponential ergodicity property of the fast component with every fixed slow component in the second equation of (2.4).

A2. $\eta:=\lambda_{1}-L_{g}>0$.

The following condition on the $Q_{1}$-Wiener process $W_{t}^{Q_{1}}$ is used to establish the strong convergence of $X^{\varepsilon}$ to $\bar{X}$.

A3. There exist constants $\alpha \in\left(1, \frac{3}{2}\right)$ and $\beta \in\left(0, \frac{1}{2}\right)$ such that

$$
\sum_{k=1}^{\infty} \alpha_{k} \lambda_{k}^{\alpha+2 \beta-1}<+\infty
$$

For any $x \in L^{2}$, denote by $D \varphi(x), D^{2} \varphi(x)$ the first and the second Frechét derivatives of the function $\varphi: L^{2} \rightarrow \mathbb{R}$, respectively. By Riesz representation theorem, we have

$$
D \varphi(x) \cdot h=\langle D \varphi(x), h\rangle, \quad D^{2} \varphi(x) \cdot(h, k)=\left\langle D^{2} \varphi(x) h, k\right\rangle, \quad h, k \in L^{2} .
$$

The following condition is used to establish the weak convergence of $X^{\varepsilon}$ to $\bar{X}$.

A4. Assume that $f$ and $g$ are twice differentiable with respect to the first and the second variable, respectively, and that there exists a constant $C>0$ such that for any $x, y, h, k \in L^{2}$, the following inequalities hold:

$$
\begin{aligned}
& \left\|D_{x x}^{2} f(x, y)(h, k)\right\| \leqslant C\|h\|\|k\| ; \\
& \left\|D_{y} g(x, y) \cdot h\right\| \leqslant C\|h\|, \quad\left|D_{y y}^{2} g(x, y)(h, k)\right| \leqslant C\|h\|\|k\| ; \\
& |\langle f(x, y), x\rangle| \leqslant C\left(1+\|x\|^{2}\right) .
\end{aligned}
$$

Now we are going to formulate our main results. The first result gives the convergence rate in the sense of the trajectory distance between the slow component $X_{t}^{\varepsilon}$ and the averaged component $\bar{X}_{t}$, as $\varepsilon \rightarrow 0$, uniformly with respect to $t \in[0, T]$.

Theorem 2.1 (Strong convergence). Assume the conditions A1, A2 and A3 hold. Then, for any $x \in H^{\alpha}$ with $\alpha$ given in $\boldsymbol{A} 3, y \in L^{2}, p>0$ and $T>0$, there exists a constant $C:=C_{x, y, T, p, \alpha}>0$ such that

$$
\mathbb{E}\left(\sup _{0 \leqslant t \leqslant T}\left\|X_{t}^{\varepsilon}-\bar{X}_{t}\right\|^{2 p}\right) \leqslant C\left(\frac{1}{-\log \varepsilon}\right)^{\frac{1}{4 p}} \rightarrow 0, \quad \text { as } \varepsilon \rightarrow 0 .
$$


With some regularity for the initial value $x$, the following result describes the convergence rate in the sense of the law distance between the slow component $X_{t}^{\varepsilon}$ and the averaged component $\bar{X}_{t}$, as $\varepsilon \rightarrow 0$.

Theorem 2.2 (Weak convergence 1). Assume the conditions A1, A2 and A4 hold, and that $Q_{1}=0$ in (2.2). Then for any $x \in H^{\theta}$ with $\theta \in(0,1], y \in L^{2}, \phi \in C_{b}^{2}\left(L^{2}\right), t \in(0, T]$, $\delta \in\left(0, \frac{1}{2}\right)$, there exists a constant $C:=C_{x, y, T, \phi, \delta}>0$ such that for any $\varepsilon \in(0,1)$, we have

$$
\left|\mathbb{E}\left[\phi\left(X_{t}^{\varepsilon}\right)\right]-\mathbb{E}\left[\phi\left(\bar{X}_{t}\right)\right]\right| \leqslant C\left(1+t^{-\theta+\frac{\theta^{2}}{1+\delta}}\right) \varepsilon^{1-r} .
$$

The constant appeared in (2.8) can become quite terse at the cost of the higher regularity condition on the initial value $x$. More precisely, we have the following result:

Theorem 2.3 (Weak convergence 2). Assume the conditions of Theorem 2.2 hold. Then, for any $x \in H^{\theta}$ with $\theta \in\left(1, \frac{3}{2}\right), y \in L^{2}, t \in(0, T]$ and $r \in(0,1)$, there exists a constant $C:=C_{x, y, T, \phi}>0$ such that

$$
\left|\mathbb{E}\left[\phi\left(X_{t}^{\varepsilon}\right)\right]-\mathbb{E}\left[\phi\left(\bar{X}_{t}\right)\right]\right| \leqslant C \varepsilon^{1-r} .
$$

Remark 2.4. In Theorems 2.2 and 2.3, if we don't impose the condition $Q_{1}=0$ in the system (2.2), there exist some essential difficulties. For instance, it is not clear how to establish Lemma 4.8, which plays a crucial role in proving Theorems 2.2 and 2.3.

\section{Proof of Theorem 2.1}

In this section, we are devoted to proving Theorem 2.1. The proof consists of the following several steps. In the first step, we give some priori estimates of the solution $\left(X_{t}^{\varepsilon}, Y_{t}^{\varepsilon}\right)$ to the system (2.2). In the second step, following the idea inspired by Khasminskii in [21], we introduce an auxiliary process $\left(\hat{X}_{t}^{\varepsilon}, \hat{Y}_{t}^{\varepsilon}\right) \in L^{2} \times L^{2}$ and also give the uniform bounds. Meanwhile, we deduce an estimate of the process $X_{t}^{\varepsilon}-\hat{X}_{t}^{\varepsilon}$ in the space $L^{2 p}\left(\Omega, C\left([0, T], L^{2}\right)\right)$. In the last step, based on the ergodicity property of the averaged equation (see (3.25)), we make use of the skill of the stopping time and some approximation techniques to give a control of $\left|\hat{X}_{t}^{\varepsilon}-\bar{X}_{t}^{\varepsilon}\right|_{L^{2 p}\left(\Omega, C\left([0, T], L^{2}\right)\right)}$. Consequently, we deduce the convergence rate in Theorem 2.1.

Recalling that $V:=H_{0}^{1}$ is continuously and densely embedded in $L^{2}$, consider the Gelfand triple: $V \subset L^{2} \subset V^{*}$, where $V^{*}$ is the dual space of $V$. According to the Poincaré inequality, we have that for any $x \in V$,

$$
V^{*}\langle A x, x\rangle_{V}=-\|\nabla x\|^{2} \leqslant-\lambda_{1}\|x\|^{2},
$$

where $V^{*}\langle\cdot, \cdot\rangle_{V}$ denotes the dualization between $V^{*}$ and $V$.

3.1. Some priori estimates of $\left(X_{t}^{\varepsilon}, Y_{t}^{\varepsilon}\right)$. We first prove the uniform bounds, with respect to $\varepsilon \in(0,1)$ and $t \in[0, T]$, for $p$-moments of the solutions to the system (2.2). The main proof follows the techniques in [28] and [25, 26], where the authors deal with the 2D stochastic Navier-Stokes equation and 1D stochastic Burgers' equation, respectively.

Lemma 3.1. Under conditions $\boldsymbol{A} 1$ and $\boldsymbol{A} \mathscr{2}$, for any $x, y \in L^{2}, p \geqslant 2$ and $T>0$, there exists a constant $C_{p, T}>0$ such that

$$
\begin{aligned}
& \sup _{\varepsilon \in(0,1)} \sup _{0 \leqslant t \leqslant T} \mathbb{E}\left\|X_{t}^{\varepsilon}\right\|^{2 p} \leqslant C_{p, T}\left(1+\|x\|^{2 p}+\|y\|^{2 p}\right), \\
& \sup _{\varepsilon \in(0,1)} \sup _{0 \leqslant t \leqslant T} \mathbb{E}\left\|Y_{t}^{\varepsilon}\right\|^{2 p} \leqslant C_{p, T}\left(1+\|x\|^{2 p}+\|y\|^{2 p}\right) .
\end{aligned}
$$


Proof. According to Itô's formula, we have

$$
\begin{aligned}
\frac{d}{d t} \mathbb{E}\left\|Y_{t}^{\varepsilon}\right\|^{2 p}= & \frac{2 p \lambda_{1}}{\varepsilon} \mathbb{E}\left[\left\|Y_{t}^{\varepsilon}\right\|^{2 p-2}\left(-\left|Y_{t}^{\varepsilon}\right|_{1}^{2}\right)\right]+\frac{2 p}{\varepsilon} \mathbb{E}\left[\left\|Y_{t}^{\varepsilon}\right\|^{2 p-2}\left\langle g\left(X_{t}^{\varepsilon}, Y_{t}^{\varepsilon}\right), Y_{t}^{\varepsilon}\right\rangle\right] \\
& +\frac{p}{\varepsilon} \mathbb{E}\left\|Y_{t}^{\varepsilon}\right\|^{2 p-2} \operatorname{Tr} Q_{2}+\frac{2 p(p-1)}{\varepsilon} \mathbb{E}\left\|Y_{t}^{\varepsilon}\right\|^{2 p-2} \operatorname{Tr} Q_{2},
\end{aligned}
$$

where Itô's formula can be understood in the way that we first use the Galerkin approximation to get (3.4) in the finite dimensional setting, then we take the limit of the dimension to obtain (3.4) in the infinite dimensional setting. Using (3.1) and condition A1, it follows from (3.4) that

$$
\begin{aligned}
\frac{d}{d t} \mathbb{E}\left\|Y_{t}^{\varepsilon}\right\|^{2 p} \leqslant & -\frac{2 p \lambda_{1}}{\varepsilon} \mathbb{E}\left\|Y_{t}^{\varepsilon}\right\|^{2 p}+\frac{2 p}{\varepsilon} \mathbb{E}\left\{\left\|Y_{t}^{\varepsilon}\right\|^{2 p-2}\left[C\left\|Y_{t}^{\varepsilon}\right\|+L_{g}\left(\left\|X_{t}^{\varepsilon}\right\|\left\|Y_{t}^{\varepsilon}\right\|+\left\|Y_{t}^{\varepsilon}\right\|^{2}\right)\right]\right\} \\
& +\frac{p}{\varepsilon} \mathbb{E}\left\|Y_{t}^{\varepsilon}\right\|^{2 p-2} \operatorname{Tr} Q_{2}+\frac{2 p(p-1)}{\varepsilon} \mathbb{E}\left\|Y_{t}^{\varepsilon}\right\|^{2 p-2} \operatorname{Tr} Q_{2} .
\end{aligned}
$$

From (3.5), using condition A2 and the Young inequality, we deduce that there exists a constant $\gamma>0$ such that

$$
\frac{d}{d t} \mathbb{E}\left\|Y_{t}^{\varepsilon}\right\|^{2 p} \leqslant-\frac{p \gamma}{\varepsilon} \mathbb{E}\left\|Y_{t}^{\varepsilon}\right\|^{2 p}+\frac{C_{p}}{\varepsilon} \mathbb{E}\left\|X_{t}^{\varepsilon}\right\|^{2 p}+\frac{C_{p}}{\varepsilon} .
$$

Applying the comparison theorem gives

$$
\mathbb{E}\left\|Y_{t}^{\varepsilon}\right\|^{2 p} \leqslant\|y\|^{2 p} e^{-\frac{p \gamma}{\varepsilon} t}+\frac{C_{p}}{\varepsilon} \int_{0}^{t} e^{-\frac{p \gamma}{\varepsilon}(t-s)}\left(1+\mathbb{E}\left\|X_{s}^{\varepsilon}\right\|^{2 p}\right) d s .
$$

For $X_{t}^{\varepsilon}$, note that by Lemma $5.1, b(x, y, y)=0$ for any $x, y \in H_{0}^{1}$. Similarly to (3.4), applying Itô's formula, we have

$$
\begin{aligned}
\frac{d}{d t} \mathbb{E}\left\|X_{t}^{\varepsilon}\right\|^{2 p}= & 2 p \lambda_{1} \mathbb{E}\left[\left\|X_{t}^{\varepsilon}\right\|^{2 p-2}\left|X_{t}^{\varepsilon}\right|_{1}^{2}\right]+2 p \mathbb{E}\left[\left\|X_{t}^{\varepsilon}\right\|^{2 p-2}\left\langle f\left(X_{t}^{\varepsilon}, Y_{t}^{\varepsilon}\right), X_{t}^{\varepsilon}\right\rangle\right] \\
& +p \mathbb{E}\left\|X_{t}^{\varepsilon}\right\|^{2 p-2} \operatorname{Tr} Q_{1}+2 p(p-1) \mathbb{E}\left\{\left\|X_{t}^{\varepsilon}\right\|^{2 p-4}\left\|\sqrt{Q_{1}} X_{t}^{\varepsilon}\right\|^{2}\right\} .
\end{aligned}
$$

Using (3.1), we obtain

$$
\begin{aligned}
\frac{d}{d t} \mathbb{E}\left\|X_{t}^{\varepsilon}\right\|^{2 p} \leqslant & -2 p \lambda_{1} \mathbb{E}\left\|X_{t}^{\varepsilon}\right\|^{2 p}+2 p \mathbb{E}\left[\left\|X_{t}^{\varepsilon}\right\|^{2 p-2}\left\langle f\left(X_{t}^{\varepsilon}, Y_{t}^{\varepsilon}\right), X_{t}^{\varepsilon}\right\rangle\right] \\
& +p \mathbb{E}\left\|X_{t}^{\varepsilon}\right\|^{2 p-2} \operatorname{Tr} Q_{1}+2 p(p-1) \mathbb{E}\left\{\left\|X_{t}^{\varepsilon}\right\|^{2 p-4}\left\|\sqrt{Q_{1}} X_{t}^{\varepsilon}\right\|^{2}\right\} .
\end{aligned}
$$

In the same way as in (3.6) and (3.7), one can verify that

$$
\mathbb{E}\left\|X_{t}^{\varepsilon}\right\|^{2 p} \leqslant\|x\|^{2 p} e^{C_{p} t}+C_{p} \int_{0}^{t} e^{C_{p}(t-s)}\left(1+\mathbb{E}\left\|Y_{s}^{\varepsilon}\right\|^{2 p}\right) d s .
$$

Combining (3.7) and (3.8), we get that, for any $t \in[0, T]$,

$$
\mathbb{E}\left\|Y_{t}^{\varepsilon}\right\|^{2 p} \leqslant C_{p, T}\left(1+\|x\|^{2 p}+\|y\|^{2 p}\right)+\frac{C_{p}}{\varepsilon} \int_{0}^{t} e^{-\frac{p \gamma}{\varepsilon}(t-s)} \int_{0}^{s} \mathbb{E}\left\|Y_{r}^{\varepsilon}\right\|^{2 p} d r d s+\frac{C_{p}}{\varepsilon} \int_{0}^{t} e^{-\frac{p \gamma}{\varepsilon}(t-s)} d s,
$$

which implies

$$
\mathbb{E}\left\|Y_{t}^{\varepsilon}\right\|^{2 p} \leqslant C_{p, T}\left(1+\|x\|^{2 p}+\|y\|^{2 p}\right)+C_{p} \int_{0}^{t} \mathbb{E}\left\|Y_{r}^{\varepsilon}\right\|^{2 p} d r
$$

Using Gronwall's inequality, we get (3.3). The inequality (3.2) follows by combining (3.3) and (3.8). The proof is complete. 
In order to estimate the high-order norm of $\left|X_{t}^{\varepsilon}\right|_{\alpha}$, with $\alpha \in\left(1, \frac{3}{2}\right)$, we first give a control of the stochastic convolution $W_{A}(t):=\int_{0}^{t} e^{(t-s) A} d W_{s}^{Q_{1}}$.

Lemma 3.2. Under the condition $\boldsymbol{A} 3$, for any $p, T>0$ and $\alpha \in\left(1, \frac{3}{2}\right)$ which is given in A3, there exists a positive constant $C_{p, T}$ such that

$$
\mathbb{E} \sup _{0 \leqslant t \leqslant T}\left|W_{A}(t)\right|_{\alpha}^{2 p} \leqslant C_{p, T}
$$

Proof. By the Hölder inequality, it suffices to prove (3.9) for large enough $p$. Using the factorization method, for $\beta \in\left(0, \frac{1}{2}\right)$ in $\mathbf{A} 3$, we write

$$
W_{A}(t)=\frac{\sin \pi \beta}{\pi} \int_{0}^{t} e^{(t-s) A}(t-s)^{\beta-1} Z_{s} d s
$$

where

$$
Z_{s}=\int_{0}^{s} e^{(s-r) A}(s-r)^{-\beta} d W_{r}^{Q_{1}}
$$

Choosing $p>1$ large enough such that $\frac{2 p(1-\beta)}{2 p-1}<1$, we get

$$
\left|W_{A}(t)\right|_{\alpha} \leqslant C\left(\int_{0}^{t}(t-s)^{-\frac{2 p(1-\beta)}{2 p-1}} d s\right)^{\frac{2 p-1}{2 p}}|Z|_{L^{2 p}\left(0, T ; H^{\alpha}\right)} \leqslant C_{p} t^{\beta-\frac{1}{2 p}}|Z|_{L^{2 p}\left(0, T ; H^{\alpha}\right)},
$$

which implies

$$
\sup _{0 \leqslant t \leqslant T}\left|W_{A}(t)\right|_{\alpha}^{2 p} \leqslant C_{p, T}|Z|_{L^{2 p}\left(0, T ; H^{\alpha}\right)}^{2 p}
$$

Notice that $(-A)^{\alpha / 2} Z_{s} \sim N\left(0, \tilde{Q}_{s}\right)$, which is a Gaussian random variable with mean zero and the covariance operator given by

$$
\tilde{Q}_{s} x=\int_{0}^{s} r^{-2 \beta} e^{r A}(-A)^{\alpha} Q_{1} e^{r A^{*}} x d r .
$$

For any $p \geqslant 1, s>0$, we follow the proof of [8, Corollary 2.17] to obtain

$$
\begin{aligned}
\mathbb{E}\left|(-A)^{\alpha / 2} Z_{s}\right|^{2 p} & \leqslant C_{p}\left[\operatorname{Tr}\left(\tilde{Q}_{s}\right)\right]^{p}=C_{p}\left(\sum_{k=1}^{\infty} \int_{0}^{s} r^{-2 \beta} e^{-2 r \lambda_{k}} \lambda_{k}^{\alpha} \alpha_{k} d r\right)^{p} \\
& =C_{p}\left(\sum_{k=1}^{\infty} \lambda_{k}^{\alpha+2 \beta-1} \alpha_{k} \int_{0}^{2 s \lambda_{k}} r^{-2 \beta} e^{-r} d r\right)^{p} \leqslant C_{p}\left(\sum_{k=1}^{\infty} \lambda_{k}^{\alpha+2 \beta-1} \alpha_{k}\right)^{p}<\infty
\end{aligned}
$$

where in the last two inequalities we use the fact $\int_{0}^{2 s \lambda_{k}} r^{-2 \beta} e^{-r} d r \leqslant \int_{0}^{\infty} r^{-2 \beta} e^{-r} d r$, and the condition A3. We conclude the proof of Lemma 3.2 by combining (3.10) and (3.11).

Lemma 3.3. Under the conditions $\boldsymbol{A} \mathbf{1}-\boldsymbol{A} 3$, for $T>0$ and $p>0$, there exists a constant $C_{p, T}>0$ such that for any $x \in H^{\alpha}$ with $\alpha$ given in $\mathbf{A 3}$, and for any $y \in L^{2}$, we have

$$
\sup _{\varepsilon \in(0,1)} \mathbb{E}\left(\sup _{t \in[0, T]}\left|X_{t}^{\varepsilon}\right|_{\alpha}^{2 p}\right) \leqslant C_{p, T}\left(1+|x|_{\alpha}^{2 p}+\|y\|^{2 p}\right)
$$

Proof. Using the Hölder inequality, it suffices to prove (3.12) for large enough $p$. Recall that

$$
X_{t}^{\varepsilon}=e^{t A} x+\int_{0}^{t} e^{(t-s) A} B\left(X_{s}^{\varepsilon}\right) d s+\int_{0}^{t} e^{(t-s) A} f\left(X_{s}^{\varepsilon}, Y_{s}^{\varepsilon}\right) d s+\int_{0}^{t} e^{(t-s) A} d W_{s}^{Q_{1}}
$$


For the first term, it is clear that $\left|e^{A t} x\right|_{\alpha}^{2 p} \leqslant|x|_{\alpha}^{2 p}$. For the second term, according to (2.1) and Lemma 5.2, we have

$$
\begin{aligned}
\left|\int_{0}^{t} e^{(t-s) A} B\left(X_{s}^{\varepsilon}\right) d s\right|_{\alpha} & \leqslant \int_{0}^{t}\left|e^{(t-s) A} B\left(X_{s}^{\varepsilon}\right)\right|_{\alpha} d s \\
& \leqslant C \int_{0}^{t}\left(1+(t-s)^{\frac{-\alpha_{3}-\alpha}{2}}\right)\left|B\left(X_{s}^{\varepsilon}\right)\right|_{-\alpha_{3}} d s \\
& \leqslant C \int_{0}^{t}\left(1+(t-s)^{\frac{-\alpha_{3}-\alpha}{2}}\right)\left|X_{s}^{\varepsilon}\right|_{\alpha_{1}}\left|X_{s}^{\varepsilon}\right|_{\alpha_{2}+1} d s
\end{aligned}
$$

where $\alpha_{1}+\alpha_{2}+\alpha_{3}>\frac{1}{2}$ and $\alpha_{i}>0, i=1,2,3$. Using the interpolation inequality, we have that

$$
\left|X_{s}^{\varepsilon}\right|_{\alpha_{1}} \leqslant C\left\|X_{s}^{\varepsilon}\right\|^{\frac{\alpha-\alpha_{1}}{\alpha}}\left|X_{s}^{\varepsilon}\right|_{\alpha^{\frac{\alpha_{1}}{\alpha}}}
$$

for any $0<\alpha_{1}<\alpha$, and that

$$
\left|X_{s}^{\varepsilon}\right|_{\alpha_{2}+1} \leqslant C\left\|X_{s}^{\varepsilon}\right\|^{\frac{\alpha-\alpha_{2}-1}{\alpha}}\left|X_{s}^{\varepsilon}\right|^{\frac{\alpha_{2}+1}{\alpha}}
$$

for any $0<\alpha_{2}+1<\alpha$. Let $\alpha_{1}$ and $\alpha_{2}$ be small enough such that $1+\alpha_{1}+\alpha_{2} \in(1, \alpha)$. It follows from (3.13) and (3.14) that

$$
\begin{aligned}
& \mathbb{E} \sup _{t \in[0, T]}\left|\int_{0}^{t} e^{(t-s) A} B\left(X_{s}^{\varepsilon}\right) d s\right|_{\alpha}^{2 p} \\
\leqslant & C \mathbb{E}\left(\sup _{t \in[0, T]} \int_{0}^{t}\left(1+(t-s)^{\frac{-\alpha_{3}-\alpha}{2}}\right)\left\|X_{s}^{\varepsilon}\right\|^{\frac{2 \alpha-\alpha_{1}-\alpha_{2}-1}{\alpha}}\left|X_{s}^{\varepsilon}\right|^{\frac{\alpha_{1}+\alpha_{2}+1}{\alpha}} d s\right)^{2 p} \\
\leqslant & C_{p, T} \mathbb{E}\left[\left(\sup _{t \in[0, T]} \int_{0}^{t}\left(1+(t-s)^{\frac{-\alpha_{3}-\alpha}{2}}\right)^{\frac{2 p}{2 p-1}} d s\right)^{2 p-1}\right. \\
& \left.\times\left(\sup _{t \in[0, T]} \int_{0}^{t}\left\|X_{s}^{\varepsilon}\right\|^{2 p \cdot \frac{2 \alpha-\alpha_{1}-\alpha_{2}-1}{\alpha}}\left|X_{s}^{\varepsilon}\right|_{\alpha}^{2 p \cdot \frac{\alpha_{1}+\alpha_{2}+1}{\alpha}} d s\right)\right] \\
\leqslant & C_{p, T}\left(1+\int_{0}^{T} s^{-\frac{\alpha_{3}+\alpha}{2} \cdot \frac{2 p}{2 p-1}} d s\right)^{2 p-1}\left(\int_{0}^{T} \mathbb{E}\left\|X_{s}^{\varepsilon}\right\|^{2 p \cdot \frac{2 \alpha-\alpha_{1}-\alpha_{2}-1}{\alpha-\alpha_{1}-\alpha_{2}-1}} d s+\int_{0}^{T} \mathbb{E}\left|X_{s}^{\varepsilon}\right|_{\alpha}^{2 p} d s\right) .
\end{aligned}
$$

By Lemma 3.1, we obtain

$$
\mathbb{E} \sup _{t \in[0, T]}\left|\int_{0}^{t} e^{(t-s) A} B\left(X_{s}^{\varepsilon}\right) d s\right|_{\alpha}^{2 p} \leqslant C_{p, T}\left(1+\int_{0}^{T} s^{-\frac{\alpha_{3}+\alpha}{2} \cdot \frac{2 p}{2 p-1}} d s\right)^{2 p-1}\left(1+\int_{0}^{T} \mathbb{E}\left|X_{s}^{\varepsilon}\right|_{\alpha}^{2 p} d s\right) .
$$

We choose positive constants $\alpha_{1}, \alpha_{2}, \alpha_{3}$ such that $0<1+\alpha_{1}+\alpha_{2}<\alpha$ and $\alpha_{1}+\alpha_{2}+\alpha_{3}>\frac{1}{2}$. Let $p$ be large enough such that $\frac{\alpha+\alpha_{3}}{2} \cdot \frac{2 p}{2 p-1}<1$ (for instance, $\alpha_{3}=\frac{1}{2}, \alpha_{1}=\alpha_{2}=\frac{\alpha-1}{4}$, and $\left.p>\frac{2}{3-2 \alpha}\right)$. Consequently, from (3.15), we get

$$
\mathbb{E}\left(\sup _{t \in[0, T]}\left|\int_{0}^{t} e^{(t-s) A} B\left(X_{s}^{\varepsilon}\right) d s\right|_{\alpha}^{2 p}\right) \leqslant C_{p, T}\left(1+\int_{0}^{T} \mathbb{E}\left|X_{s}^{\varepsilon}\right|_{\alpha}^{2 p} d s\right)
$$


For the third term, according to (2.1), we obtain

$$
\begin{aligned}
& \mathbb{E}\left(\sup _{t \in[0, T]}\left|\int_{0}^{t} e^{(t-s) A} f\left(X_{s}^{\varepsilon}, Y_{s}^{\varepsilon}\right) d s\right|_{\alpha}^{2 p}\right) \\
\leqslant & C \mathbb{E}\left[\sup _{t \in[0, T]} \int_{0}^{t}\left(1+(t-s)^{-\frac{\alpha}{2}}\right)\left(1+\left\|X_{s}^{\varepsilon}\right\|+\left\|Y_{s}^{\varepsilon}\right\|\right) d s\right]^{2 p} \\
\leqslant & C_{p, T}\left(1+\int_{0}^{T} s^{-\frac{\alpha p}{2 p-1}} d s\right)^{2 p-1} \int_{0}^{T}\left(1+\mathbb{E}\left\|X_{s}^{\varepsilon}\right\|^{2 p}+\mathbb{E}\left\|Y_{s}^{\varepsilon}\right\|^{2 p}\right) d s .
\end{aligned}
$$

Taking $p$ large enough such that $\frac{\alpha p}{2 p-1}<1$, it follows from Lemma 3.1 that

$$
\mathbb{E}\left(\sup _{t \in[0, T]}\left|\int_{0}^{t} e^{(t-s) A} f\left(X_{s}^{\varepsilon}, Y_{s}^{\varepsilon}\right) d s\right|_{\alpha}^{2 p}\right) \leqslant C_{p, T}\left(1+\|x\|^{2 p}+\|y\|^{2 p}\right) .
$$

We conclude the proof of Lemma 3.3 by combining (3.16), (3.17), Lemma 3.2 and Gronwall's inequality.

Now we are equipped to prove the Hölder continuity of $t \mapsto X_{t}^{\varepsilon}$, which holds uniformly with respect to $\varepsilon \in(0,1)$.

Lemma 3.4. Under the conditions A1-A3, for any $x \in H^{\alpha}, y \in L^{2}, T>0,0<t \leqslant t+h \leqslant$ $T$, there exists a constant $C_{p, T}>0$ such that

$$
\sup _{\varepsilon \in(0,1)} \mathbb{E}\left\|X_{t+h}^{\varepsilon}-X_{t}^{\varepsilon}\right\|^{2 p} \leqslant C_{p, T} h^{p}\left(1+|x|_{\alpha}^{4 p}+\|y\|^{4 p}\right) .
$$

Proof. After simple calculations, we have

$$
\begin{aligned}
X_{t+h}^{\varepsilon}-X_{t}^{\varepsilon}= & \left(e^{A h}-I\right) X_{t}^{\varepsilon}+\int_{t}^{t+h} e^{(t+h-s) A} B\left(X_{s}^{\varepsilon}\right) d s \\
& +\int_{t}^{t+h} e^{(t+h-s) A} f\left(X_{s}^{\varepsilon}, Y_{s}^{\varepsilon}\right) d s+\int_{t}^{t+h} e^{(t+h-s) A} d W_{s}^{Q_{1}} \\
:= & I_{1}+I_{2}+I_{3}+I_{4} .
\end{aligned}
$$

For $I_{1}$, note that for $\alpha$ given in A3, there exists a constant $C_{\alpha}>0$ such that for any $x \in \mathscr{D}\left((-A)^{\frac{\alpha}{2}}\right),\left\|e^{A h} x-x\right\| \leqslant C_{\alpha} h^{\frac{\alpha}{2}}|x|_{\alpha}$. Then using Lemma 3.3, we get

$$
\mathbb{E}\left\|I_{1}\right\|^{2 p} \leqslant C_{\alpha} h^{\alpha p} \mathbb{E}\left|X_{t}^{\varepsilon}\right|_{\alpha}^{2 p} \leqslant C_{p, T} h^{\alpha p}\left(1+|x|_{\alpha}^{2 p}+\|y\|^{2 p}\right) .
$$

For $I_{2}$, using the contractive property of the semigroup $e^{t A}$, Corollary 5.3 and Lemma 3.3, we obtain

$$
\begin{aligned}
\mathbb{E}\left\|I_{2}\right\|^{2 p} & \leqslant \mathbb{E}\left(\int_{t}^{t+h}\left\|B\left(X_{s}^{\varepsilon}\right)\right\| d s\right)^{2 p} \leqslant C \mathbb{E}\left(\int_{t}^{t+h}\left|X_{s}^{\varepsilon}\right|_{1}^{2} d s\right)^{2 p} \\
& \leqslant C_{p, T} h^{2 p} \mathbb{E} \sup _{s \in[0, T]}\left|X_{s}^{\varepsilon}\right|_{1}^{4 p} \leqslant C_{p, T} h^{2 p}\left(1+|x|_{\alpha}^{4 p}+\|y\|^{4 p}\right) .
\end{aligned}
$$

For $I_{3}$, applying condition $\mathbf{A} 1$ and Lemma 3.1, we get

$$
\begin{aligned}
\mathbb{E}\left\|I_{3}\right\|^{2 p} & \leqslant h^{2 p-1} \mathbb{E} \int_{t}^{t+h}\left\|f\left(X_{s}^{\varepsilon}, Y_{s}^{\varepsilon}\right)\right\|^{2 p} d s \\
& \leqslant C h^{2 p-1} \mathbb{E} \int_{t}^{t+h}\left(1+\left\|X_{s}^{\varepsilon}\right\|+\left\|Y_{s}^{\varepsilon}\right\|\right)^{2 p} d s \leqslant C_{p, T} h^{2 p}\left(1+\|x\|^{2 p}+\|y\|^{2 p}\right) .
\end{aligned}
$$


For $I_{4}$, note that $I_{4}$ is the centered Gaussian random variable with the variance given by $S_{h} x=\int_{0}^{h} e^{(h-r) A} Q_{1} e^{(h-r) A^{*}} x d r$. Then, for any $p \geqslant 1$, we get

$$
\mathbb{E}\left\|I_{4}\right\|^{2 p} \leqslant C_{p}\left[\operatorname{Tr}\left(S_{h}\right)\right]^{p}=C_{p}\left(\sum_{k=1}^{\infty} \int_{0}^{h} e^{-2(h-r) \lambda_{k}} \alpha_{k} d r\right)^{p} \leqslant C_{p}\left(\sum_{k=1}^{\infty} \alpha_{k}\right)^{p} h^{p} .
$$

Putting (3.18)-(3.21) together, the result follows.

3.2. Estimates of the auxiliary process $\left(\hat{X}_{t}^{\varepsilon}, \hat{Y}_{t}^{\varepsilon}\right)$. Following the idea inspired by Khasminskii [21], we introduce an auxiliary process $\left(\hat{X}_{t}^{\varepsilon}, \hat{Y}_{t}^{\varepsilon}\right) \in L^{2} \times L^{2}$. Specifically, we split the interval $[0, T]$ into some subintervals of size $\delta>0$. With the initial value $\hat{Y}_{0}^{\varepsilon}=Y_{0}^{\varepsilon}=y$, for any $t \in[k \delta, \min ((k+1) \delta, T)), k \in \mathbb{N}$, we construct the process $\hat{Y}_{t}^{\varepsilon}$ as follows:

$$
\hat{Y}_{t}^{\varepsilon}=Y_{k \delta}^{\varepsilon}+\frac{1}{\varepsilon} \int_{k \delta}^{t} A \hat{Y}_{s}^{\varepsilon} d s+\frac{1}{\varepsilon} \int_{k \delta}^{t} g\left(X_{k \delta}^{\varepsilon}, \hat{Y}_{s}^{\varepsilon}\right) d s+\frac{1}{\sqrt{\varepsilon}} \int_{k \delta}^{t} d W_{s}^{Q_{2}},
$$

where $\left(X_{s}^{\varepsilon}, Y_{s}^{\varepsilon}\right)$ is the solution to the system (2.2). Then, for any $t \in[0, T]$, we construct the process $\hat{X}_{t}^{\varepsilon}$ as follows:

$$
\hat{X}_{t}^{\varepsilon}=x+\int_{0}^{t} A \hat{X}_{s}^{\varepsilon} d s+\int_{0}^{t} B\left(X_{s(\delta)}^{\varepsilon}\right) d s+\int_{0}^{t} f\left(X_{s(\delta)}^{\varepsilon}, \hat{Y}_{s}^{\varepsilon}\right) d s+W_{t}^{Q_{1}}
$$

where $s(\delta)=\left[\frac{s}{\delta}\right] \delta$ is the nearest breakpoint proceeding $s$. Note that for any $t \in[k \delta,(k+1) \delta)$, the fast component $\hat{Y}_{t}^{\varepsilon}$ does not depend on the slow component $\hat{X}_{t}^{\varepsilon}$. The following result gives a control of the auxiliary process $\left(\hat{X}_{t}^{\varepsilon}, \hat{Y}_{t}^{\varepsilon}\right)$.

Lemma 3.5. Under the conditions $\boldsymbol{A} 1$ - $\boldsymbol{A}$ 3, for any $x, y \in L^{2}, p \geqslant 2$ and $T>0$, there exists a constant $C_{p, T}>0$ such that

$$
\sup _{\varepsilon \in(0,1)} \sup _{t \in[0, T]} \mathbb{E}\left\|\hat{Y}_{t}^{\varepsilon}\right\|^{2 p} \leqslant C_{p, T}\left(1+\|x\|^{2 p}+\|y\|^{2 p}\right)
$$

In addition, for any $x \in H^{\alpha}, y \in L^{2}, p \geqslant 2$ and $T>0$, we have

$$
\sup _{\varepsilon \in(0,1)} \mathbb{E}\left(\sup _{t \in[0, T]}\left|\hat{X}_{t}^{\varepsilon}\right|_{\alpha}^{2 p}\right) \leqslant C_{p, T}\left(1+|x|_{\alpha}^{2 p}+\|y\|^{2 p}\right)
$$

From the construction of $\left(\hat{X}_{t}^{\varepsilon}, \hat{Y}_{t}^{\varepsilon}\right)$, since the proof of Lemma 3.5 can be carried out in the same way as in the proof of Lemmas 3.1 and 3.3, we omit the details.

We now give a control of $Y_{t}^{\varepsilon}-\hat{Y}_{t}^{\varepsilon}$.

Lemma 3.6. Under the conditions $\boldsymbol{A} 1$ - $\boldsymbol{A} 3$, for any $x \in H^{\alpha}, y \in L^{2}, p \geqslant 2, T>0$ and $\varepsilon \in(0,1)$, there exists a constant $C_{p, T}>0$ such that

$$
\sup _{0 \leqslant t \leqslant T} \mathbb{E}\left\|Y_{t}^{\varepsilon}-\hat{Y}_{t}^{\varepsilon}\right\|^{2 p} \leqslant C_{p, T}\left(1+|x|_{\alpha}^{4 p}+\|y\|^{4 p}\right) \frac{\delta^{p+1}}{\varepsilon}
$$


Proof. For $t \in[0, T]$ with $t \in[k \delta,(k+1) \delta)$, by Itô's formula and Lemma 3.4, similarly to (3.4), we have

$$
\begin{aligned}
\frac{d}{d t} \mathbb{E}\left\|Y_{t}^{\varepsilon}-\hat{Y}_{t}^{\varepsilon}\right\|^{2 p}= & \frac{2 p}{\varepsilon} \mathbb{E}\left[\left\|Y_{t}^{\varepsilon}-\hat{Y}_{t}^{\varepsilon}\right\|^{2 p-2}\left(-\left|Y_{t}^{\varepsilon}-\hat{Y}_{t}^{\varepsilon}\right|_{1}^{2}\right)\right] \\
& +\frac{2 p}{\varepsilon} \mathbb{E}\left[\left\|Y_{t}^{\varepsilon}-\hat{Y}_{t}^{\varepsilon}\right\|^{2 p-2}\left\langle g\left(X_{t}^{\varepsilon}, Y_{t}^{\varepsilon}\right)-g\left(X_{k \delta}^{\varepsilon}, \hat{Y}_{t}^{\varepsilon}\right),\left(Y_{t}^{\varepsilon}-\hat{Y}_{t}^{\varepsilon}\right)\right\rangle\right] \\
\leqslant & -\frac{2 p}{\varepsilon}\left(\lambda_{1}-L_{g}\right) \mathbb{E}\left[\left\|Y_{t}^{\varepsilon}-\hat{Y}_{t}^{\varepsilon}\right\|^{2 p}\right] \\
& +\frac{p}{\varepsilon}\left(\lambda_{1}-L_{g}\right) \mathbb{E}\left[\left\|Y_{t}^{\varepsilon}-\hat{Y}_{t}^{\varepsilon}\right\|^{2 p}\right]+\frac{C_{p}}{\varepsilon} \mathbb{E}\left[\left\|X_{t}^{\varepsilon}-X_{k \delta}^{\varepsilon}\right\|^{2 p}\right] \\
\leqslant & -\frac{p}{\varepsilon}\left(\lambda_{1}-L_{g}\right) \mathbb{E}\left\|Y_{t}^{\varepsilon}-\hat{Y}_{t}^{\varepsilon}\right\|^{2 p}+C_{p, T}\left(1+|x|_{\alpha}^{4 p}+\|y\|^{4 p}\right) \frac{(t-k \delta)^{p}}{\varepsilon} .
\end{aligned}
$$

Gronwall's inequality yields that

$$
\begin{aligned}
\mathbb{E}\left\|Y_{t}^{\varepsilon}-\hat{Y}_{t}^{\varepsilon}\right\|^{2 p} & \leqslant \frac{C_{p, T}}{\varepsilon}\left(1+|x|_{\alpha}^{4 p}+\|y\|^{4 p}\right) \int_{k \delta}^{t} e^{-\frac{p}{\varepsilon}\left(\lambda_{1}-L_{g}\right)(t-s)}(s-k \delta)^{p} d s \\
& \leqslant C_{p, T}\left(1+|x|_{\alpha}^{4 p}+\|y\|^{4 p}\right) \frac{\delta^{p+1}}{\varepsilon},
\end{aligned}
$$

which ends the proof.

Lemma 3.7. Under the conditions $\boldsymbol{A} 1$ - $\boldsymbol{A} 3$, for any $x \in H^{\alpha}, y \in L^{2}, p \geqslant 2, T>0$ and $\varepsilon \in(0,1)$, there exists a constant $C_{p, T}>0$ such that

$$
\mathbb{E}\left(\sup _{0 \leqslant t \leqslant T}\left\|X_{t}^{\varepsilon}-\hat{X}_{t}^{\varepsilon}\right\|^{2 p}\right) \leqslant C_{p, T}\left(\delta^{p}+\frac{\delta^{p+1}}{\varepsilon}\right)\left(1+|x|_{\alpha}^{6 p}+\|y\|^{6 p}\right) .
$$

Proof. In view of (2.4) and (3.23), we write

$$
X_{t}^{\varepsilon}-\hat{X}_{t}^{\varepsilon}=\int_{0}^{t} e^{(t-s) A}\left[B\left(X_{s}^{\varepsilon}\right)-B\left(X_{s(\delta)}^{\varepsilon}\right)\right] d s+\int_{0}^{t} e^{(t-s) A}\left[f\left(X_{s}^{\varepsilon}, Y_{s}^{\varepsilon}\right)-f\left(X_{s(\delta)}^{\varepsilon}, \hat{Y}_{s}^{\varepsilon}\right)\right] d s .
$$

Using (2.1), condition A1 and Lemma 5.4, we get

$$
\begin{aligned}
\left\|X_{t}^{\varepsilon}-\hat{X}_{t}^{\varepsilon}\right\|^{2 p} \leqslant & C_{p}\left\{\int_{0}^{t}\left[1+(t-s)^{-\frac{1}{2}}\right]\left|B\left(X_{s}^{\varepsilon}\right)-B\left(X_{s(\delta)}^{\varepsilon}\right)\right|_{-1} d s\right\}^{2 p} \\
& +C_{p}\left\{\int_{0}^{t}\left(\left\|X_{s}^{\varepsilon}-X_{s(\delta)}^{\varepsilon}\right\|+\left\|Y_{s}^{\varepsilon}-\hat{Y}_{s}^{\varepsilon}\right\|\right) d s\right\}^{2 p} \\
\leqslant & C_{p}\left\{\int_{0}^{t}\left[1+(t-s)^{-\frac{1}{2}}\right]\left\|X_{s}^{\varepsilon}-X_{s(\delta)}^{\varepsilon}\right\|\left(\left|X_{s}^{\varepsilon}\right|_{1}+\left|X_{s(\delta)}^{\varepsilon}\right| 1\right) d s\right\}^{2 p} \\
& +C_{p, T} \int_{0}^{t}\left(\left\|X_{s}^{\varepsilon}-X_{s(\delta)}^{\varepsilon}\right\|^{2 p}+\left\|Y_{s}^{\varepsilon}-\hat{Y}_{s}^{\varepsilon}\right\|^{2 p}\right) d s \\
\leqslant & C_{p}\left\{\int_{0}^{t}\left[1+(t-s)^{-\frac{1}{2}}\right]^{\frac{2 p}{2 p-1}} d s\right\}^{2 p-1} \cdot\left\{\int_{0}^{t}\left\|X_{s}^{\varepsilon}-X_{s(\delta)}^{\varepsilon}\right\|^{4 p} d s\right\}^{\frac{1}{2}} \\
& \times\left(\int_{0}^{t}\left(\left|X_{s}^{\varepsilon}\right|_{1}+\left|X_{s(\delta)}^{\varepsilon}\right|_{1}\right)^{4 p} d s\right)^{\frac{1}{2}} \\
& +C_{p, T} \int_{0}^{t}\left(\left\|X_{s}^{\varepsilon}-X_{s(\delta)}^{\varepsilon}\right\|^{2 p}+\left\|Y_{s}^{\varepsilon}-\hat{Y}_{s}^{\varepsilon}\right\|^{2 p}\right) d s
\end{aligned}
$$


According to Lemmas 3.3, 3.4 and 3.6, we obtain

$$
\begin{aligned}
\mathbb{E}\left(\sup _{0 \leqslant t \leqslant T}\left\|X_{t}^{\varepsilon}-\hat{X}_{t}^{\varepsilon}\right\|^{2 p}\right) \leqslant & C_{p, T}\left(\int_{0}^{T} \mathbb{E}\left\|X_{s}^{\varepsilon}-X_{s(\delta)}^{\varepsilon}\right\|^{4 p} d s\right)^{\frac{1}{2}} \\
& \times\left(\int_{0}^{T}\left(\mathbb{E}\left|X_{s}^{\varepsilon}\right|_{1}^{4 p}+\mathbb{E}\left|X_{s(\delta)}^{\varepsilon}\right|_{1}^{4 p}\right) d s\right)^{\frac{1}{2}} \\
& +C_{p, T} \int_{0}^{T} \mathbb{E}\left\|X_{s}^{\varepsilon}-X_{s(\delta)}^{\varepsilon}\right\|^{2 p}+\mathbb{E}\left\|Y_{s}^{\varepsilon}-\hat{Y}_{s}^{\varepsilon}\right\|^{2 p} d s \\
\leqslant & C_{p, T}\left(\delta^{p}+\frac{\delta^{p+1}}{\varepsilon}\right)\left(1+|x|_{\alpha}^{6 p}+\|y\|^{6 p}\right) .
\end{aligned}
$$

The proof is complete.

3.3. The averaged equation. For any fixed $x \in L^{2}$, we consider the following frozen equation associated with the fast component:

$$
\left\{\begin{array}{l}
\frac{\partial Y_{t}(\xi)}{\partial t}=A Y_{t}(\xi)+g\left(x, Y_{t}(\xi)\right)+\frac{\partial W^{Q_{2}}}{\partial t}(t, \xi), Y_{0}(\xi)=y \\
Y_{t}(0)=Y_{t}(1)=0, \quad t \in[0, \infty) .
\end{array}\right.
$$

Since $g(x, \cdot)$ is Lipshcitz continuous, it is easy to prove that for any fixed $x, y \in L^{2}$, the equation (3.25) has a unique mild solution denoted by $Y_{t}^{x, y}$. For any $x \in L^{2}$, let $P_{t}^{x}$ be the transition semigroup of $Y_{t}^{x, y}$, that is, for any bounded measurable function $\varphi$ on $L^{2}$ and $t \geqslant 0$,

$$
P_{t}^{x} \varphi(y)=\mathbb{E} \varphi\left(Y_{t}^{x, y}\right), \quad y \in L^{2} .
$$

The asymptotic behavior of $P_{t}^{x}$ has been studied in many literatures. The following result shows the existence and uniqueness of the invariant measure and gives the exponential convergence to the equilibrium (see [5, Theorem 3.5]).

Proposition 3.8. For any $x, y \in L^{2}$, there exists a unique invariant measure $\mu^{x}$ for (3.25). Moreover, there exists $C>0$ such that for any bounded measurable function $\varphi: L^{2} \rightarrow \mathbb{R}$,

$$
\left|P_{t}^{x} \varphi(y)-\int_{L^{2}} \varphi(z) \mu^{x}(d z)\right| \leqslant C(1+\|x\|+\|y\|) e^{-\frac{\left(\lambda_{1}-L_{g}\right) t}{2}}(t \wedge 1)^{-1 / 2}|\varphi|_{\infty},
$$

where $|\varphi|_{\infty}=\sup _{x \in L^{2}}|\varphi(x)|$.

Furthermore, we have the following result, whose proof can refer to [5, Remark 3.6].

Proposition 3.9. For any $x, y \in L^{2}$, there exists $C>0$ such that for any Lipschitz function $\varphi: L^{2} \rightarrow \mathbb{R}$

$$
\left|P_{t}^{x} \varphi(y)-\int_{L^{2}} \varphi(z) \mu^{x}(d z)\right| \leqslant C(1+\|x\|+\|y\|) e^{-\frac{\left(\lambda_{1}-L_{g}\right) t}{2}}|\varphi|_{L i p},
$$

where $|\varphi|_{\text {Lip }}=\sup _{x, y \in L^{2}, x \neq y} \frac{|\varphi(x)-\varphi(y)|}{\|x-y\|}$.

In the sequel we shall prove that the slow component $X_{t}^{\varepsilon}$ in the system (2.2) converges strongly to $\bar{X}_{t}$, which is the solution of the averaged equation:

$$
\left\{\begin{array}{l}
d \bar{X}_{t}=A \bar{X}_{t} d t+B\left(\bar{X}_{t}\right) d t+\bar{f}\left(\bar{X}_{t}\right) d t+d W_{t}^{Q_{1}}, \\
\bar{X}_{0}=x
\end{array}\right.
$$


where

$$
\bar{f}(x)=\int_{L^{2}} f(x, y) \mu^{x}(d y), \quad x \in L^{2} .
$$

The following result gives a control of $\left|\hat{X}_{t}^{\varepsilon}-\bar{X}_{t}\right|$.

Lemma 3.10. Under the conditions A1-A3, for any $x \in H^{\alpha}, y \in L^{2}, p \geqslant 1, T>0$ and $\varepsilon \in(0,1)$, there exists a constant $C_{p, T}>0$ such that

$$
\mathbb{E} \sup _{0 \leqslant t \leqslant T}\left\|\hat{X}_{t}^{\varepsilon}-\bar{X}_{t}\right\|^{2 p} \leqslant C_{p, T}\left(1+|x|_{\alpha}^{6 p}+\|y\|^{6 p}\right)\left(\frac{1}{-\log \varepsilon}\right)^{\frac{1}{4 p}}
$$

Proof. From (3.23) and (3.26), we have

$$
\begin{aligned}
\hat{X}_{t}^{\varepsilon}-\bar{X}_{t}= & \int_{0}^{t} e^{(t-s) A}\left[B\left(X_{s(\delta)}^{\varepsilon}\right)-B\left(X_{s}^{\varepsilon}\right)\right] d s+\int_{0}^{t} e^{(t-s) A}\left[B\left(X_{s}^{\varepsilon}\right)-B\left(\hat{X}_{s}^{\varepsilon}\right)\right] d s \\
& +\int_{0}^{t} e^{(t-s) A}\left[B\left(\hat{X}_{s}^{\varepsilon}\right)-B\left(\bar{X}_{s}\right)\right] d s+\int_{0}^{t} e^{(t-s) A}\left[f\left(X_{s(\delta)}^{\varepsilon}, \hat{Y}_{s}^{\varepsilon}\right)-\bar{f}\left(X_{s}^{\varepsilon}\right)\right] d s \\
& +\int_{0}^{t} e^{(t-s) A}\left[\bar{f}\left(X_{s}^{\varepsilon}\right)-\bar{f}\left(\hat{X}_{s}^{\varepsilon}\right)\right] d s+\int_{0}^{t} e^{(t-s) A}\left[\bar{f}\left(\hat{X}_{s}^{\varepsilon}\right)-\bar{f}\left(\bar{X}_{s}\right)\right] d s \\
:= & \sum_{k=1}^{6} J_{k}(t) .
\end{aligned}
$$

For $J_{1}(t)$, in the same way as in the proof of Lemma 3.7, we get

$$
\begin{aligned}
\mathbb{E} \sup _{0 \leqslant t \leqslant T}\left\|J_{1}(t)\right\|^{2 p} & \leqslant C_{p, T}\left[\int_{0}^{T} \mathbb{E}\left\|X_{s}^{\varepsilon}-X_{s(\delta)}^{\varepsilon}\right\|^{4 p} d s\right]^{\frac{1}{2}}\left[\int_{0}^{T}\left(\mathbb{E}\left|X_{s}^{\varepsilon}\right|_{1}+\mathbb{E}\left|X_{s(\delta)}^{\varepsilon}\right|\right)^{4 p} d s\right]^{\frac{1}{2}} \\
& \leqslant C_{p, T} \delta^{p}\left(1+|x|_{\alpha}^{6 p}+\|y\|^{6 p}\right) .
\end{aligned}
$$

For $J_{2}(t)$, using Lemmas 3.3, 3.5 and 3.7 gives

$$
\begin{aligned}
\mathbb{E} \sup _{0 \leqslant t \leqslant T}\left\|J_{2}(t)\right\|^{2 p} & \leqslant C_{p, T}\left[\int_{0}^{T} \mathbb{E}\left\|X_{s}^{\varepsilon}-\hat{X}_{s}^{\varepsilon}\right\|^{4 p} d s\right]^{\frac{1}{2}}\left[\int_{0}^{T}\left(\mathbb{E}\left|X_{s}^{\varepsilon}\right|_{1}^{4 p}+\mathbb{E}\left|\hat{X}_{s}^{\varepsilon}\right|_{1}^{4 p}\right) d s\right]^{\frac{1}{2}} \\
& \leqslant C_{p, T}\left(\delta^{p}+\frac{\delta^{p+\frac{1}{2}}}{\sqrt{\varepsilon}}\right)\left(1+|x|_{\alpha}^{6 p}+\|y\|^{6 p}\right) .
\end{aligned}
$$

For $J_{3}(t)$, according to $(2.1)$ and Lemma 5.4, we have

$$
\begin{aligned}
\sup _{0 \leqslant t \leqslant T}\left\|J_{3}(t)\right\|^{2 p} & \leqslant C_{p}\left\{\sup _{0 \leqslant t \leqslant T} \int_{0}^{t}\left[1+(t-s)^{-\frac{1}{2}}\right]\left|B\left(\hat{X}_{s}^{\varepsilon}\right)-B\left(\bar{X}_{s}\right)\right|_{-1} d s\right\}^{2 p} \\
& \leqslant C_{p}\left\{\sup _{0 \leqslant t \leqslant T} \int_{0}^{t}\left[1+(t-s)^{-\frac{1}{2}}\right]\left\|\hat{X}_{s}^{\varepsilon}-\bar{X}_{s}\right\|\left(\left|\hat{X}_{s}^{\varepsilon}\right|_{1}+\left|\bar{X}_{s}\right|_{1}\right) d s\right\}^{2 p} .
\end{aligned}
$$

In order to control $J_{3}(t)$, we make a use of the skill of stopping times. For any fixed $n \geqslant 1$ and $\varepsilon>0$, define the stopping time:

$$
\tau_{n}^{\varepsilon}=\inf \left\{t>0:\left|\hat{X}_{t}^{\varepsilon}\right|_{1}+\left|\bar{X}_{t}\right|_{1}>n\right\} .
$$


It follows from (3.29) and (3.30) that

$$
\begin{aligned}
\mathbb{E} \sup _{0 \leqslant t \leqslant T \wedge \tau_{n}^{\varepsilon}}\left\|J_{3}(t)\right\|^{2 p} & \leqslant C_{p} \mathbb{E}\left(\sup _{0 \leqslant t \leqslant T \wedge \tau_{n}^{\varepsilon}} \int_{0}^{t}\left(1+(t-r)^{-\frac{1}{2}}\right)\left\|\hat{X}_{r}^{\varepsilon}-\bar{X}_{r}\right\|\left(\left|\hat{X}_{r}^{\varepsilon}\right|_{1}+\left|\bar{X}_{r}\right|_{1}\right) d r\right)^{2 p} \\
& \leqslant C_{p} n^{2 p} \mathbb{E}\left(\sup _{0 \leqslant t \leqslant T \wedge \tau_{n}^{\varepsilon}} \int_{0}^{t}\left(1+(t-r)^{-\frac{1}{2}}\right)\left\|\hat{X}_{r}^{\varepsilon}-\bar{X}_{r}\right\| d r\right)^{2 p} \\
& \leqslant C_{p} n^{2 p}\left(\sup _{0 \leqslant t \leqslant T} \int_{0}^{t}\left(1+(t-r)^{-\frac{1}{2}}\right)^{\frac{2 p}{2 p-1}} d r\right)^{2 p-1} \mathbb{E} \int_{0}^{T \wedge \tau_{n}^{\varepsilon}}\left\|\hat{X}_{r}^{\varepsilon}-\bar{X}_{r}\right\|^{2 p} d r \\
& \leqslant C_{p, T} n^{2 p} \int_{0}^{T} \mathbb{E} \sup _{0 \leqslant r \leqslant s \wedge \tau_{n}^{\varepsilon}}\left\|\hat{X}_{r}^{\varepsilon}-\bar{X}_{r}\right\|^{2 p} d s .
\end{aligned}
$$

For $J_{5}(t)$, using the contractive property of the semigroup $e^{t A}, t \geqslant 0$, Lipschitz continuity of $\bar{f}$, and Lemma 3.7, we obtain

$$
\mathbb{E} \sup _{0 \leqslant t \leqslant T}\left|J_{5}(t)\right|^{2 p} \leqslant C_{p, T} \mathbb{E} \int_{0}^{T}\left\|X_{s}^{\varepsilon}-\hat{X}_{s}\right\|^{2 p} d s \leqslant C_{p, T}\left(\delta^{p}+\frac{\delta^{p+1}}{\varepsilon}\right)\left(1+|x|_{\alpha}^{2 p}+\|y\|^{2 p}\right) .
$$

For $J_{6}(t)$, similarly to the estimate of $J_{5}(t)$, we get

$$
\mathbb{E} \sup _{0 \leqslant t \leqslant T \wedge \tau_{n}^{\varepsilon}}\left|J_{6}(t)\right|^{2 p} \leqslant C_{p, T} \int_{0}^{T} \mathbb{E} \sup _{0 \leqslant r \leqslant s \wedge \tau_{n}^{\varepsilon}}\left\|\hat{X}_{r}^{\varepsilon}-\bar{X}_{r}\right\|^{2 p} d s .
$$

For $J_{4}(t)$, set $n_{t}=\left[\frac{t}{\delta}\right]$, where $t \in[0, T)$ and $\delta>0$. We write

$$
J_{4}(t)=J_{4}^{1}(t)+J_{4}^{2}(t)+J_{4}^{3}(t),
$$

where

$$
\begin{gathered}
J_{4}^{1}(t)=\sum_{k=0}^{n_{t}-1} \int_{k \delta}^{(k+1) \delta} e^{(t-s) A}\left[f\left(X_{k \delta}^{\varepsilon}, \hat{Y}_{s}^{\varepsilon}\right)-\bar{f}\left(X_{k \delta}^{\varepsilon}\right)\right] d s, \\
J_{4}^{2}(t)=\sum_{k=0}^{n_{t}-1} \int_{k \delta}^{(k+1) \delta} e^{(t-s) A}\left[\bar{f}\left(X_{k \delta}^{\varepsilon}\right)-\bar{f}\left(X_{s}^{\varepsilon}\right)\right] d s \\
J_{4}^{3}(t)=\int_{n_{t} \delta}^{t} e^{(t-s) A}\left[f\left(X_{n_{t} \delta}^{\varepsilon}, \hat{Y}_{s}^{\varepsilon}\right)-\bar{f}\left(X_{s}^{\varepsilon}\right)\right] d s .
\end{gathered}
$$

For $J_{4}^{2}(t)$, we have

$$
\mathbb{E} \sup _{0 \leqslant t \leqslant T}\left\|J_{4}^{2}(t)\right\|^{2 p} \leqslant C_{p, T} \int_{0}^{T} \mathbb{E}\left\|X_{s(\delta)}^{\varepsilon}-X_{s}^{\varepsilon}\right\|^{2 p} d s \leqslant C_{p, T} \delta^{p}\left(1+|x|_{\alpha}^{2 p}+\|y\|^{2 p}\right) .
$$

For $J_{4}^{3}(t)$, it follows from Lemmas 3.1 and 3.5 that

$$
\begin{aligned}
\mathbb{E} \sup _{0 \leqslant t \leqslant T}\left\|J_{4}^{3}(t)\right\|^{2 p} & \leqslant C_{p} \delta^{2 p-1} \mathbb{E}\left[\sup _{0 \leqslant t \leqslant T} \int_{n_{t} \delta}^{t}\left(1+\left\|X_{n_{t} \delta}^{\varepsilon}\right\|^{2 p}+\left\|\hat{Y}_{s}^{\varepsilon}\right\|^{2 p}+\left\|X_{s}^{\varepsilon}\right\|^{2 p}\right) d s\right] \\
& \leqslant C_{p, T} \delta^{2 p-1}\left(1+\|x\|^{2 p}+\|y\|^{2 p}\right) .
\end{aligned}
$$

For $J_{4}^{1}(t)$, from the construction of $\hat{Y}_{t}^{\varepsilon}$, we obtain that, for any $k$ and $s \in[0, \delta)$,

$$
\hat{Y}_{s+k \delta}^{\varepsilon}=Y_{k \delta}^{\varepsilon}+\frac{1}{\varepsilon} \int_{0}^{s} A \hat{Y}_{r+k \delta}^{\varepsilon} d r+\frac{1}{\varepsilon} \int_{0}^{s} g\left(X_{k \delta}^{\varepsilon}, \hat{Y}_{r+k \delta}^{\varepsilon}\right) d r+\frac{1}{\sqrt{\varepsilon}} \int_{0}^{s} d \tilde{W}^{Q_{2}}(r),
$$


where $\tilde{W}^{Q_{2}}(t):=W^{Q_{2}}(t+k \delta)-W^{Q_{2}}(k \delta)$ is the shift version of $W^{Q_{2}}(t)$. Let $\bar{W}^{Q_{2}}(t)$ be a $Q_{2}$-Wiener process which is independent of $W^{Q_{1}}(t)$ and $W^{Q_{2}}(t)$. Denote by $\overline{\bar{W}}^{Q_{2}}(t)=$ $\sqrt{\varepsilon} \bar{W}^{Q_{2}}\left(\frac{t}{\varepsilon}\right)$. We construct a process $Y^{X_{k \delta}^{\varepsilon}, Y_{k \delta}^{\varepsilon}}$ by means of

$$
\begin{aligned}
Y_{\frac{s}{\varepsilon}}^{X_{k \delta}^{\varepsilon}, Y_{k \delta}^{\varepsilon}} & =Y_{k \delta}^{\varepsilon}+\int_{0}^{\frac{s}{\varepsilon}} A Y_{r}^{X_{k \delta}^{\varepsilon}, Y_{k \delta}^{\varepsilon}} d r+\int_{0}^{\frac{s}{\varepsilon}} g\left(X_{k \delta}^{\varepsilon}, Y_{r}^{X_{k \delta}^{\varepsilon}, Y_{k \delta}^{\varepsilon}}\right) d r+\int_{0}^{\frac{s}{\varepsilon}} d \bar{W}^{Q_{2}}(r) \\
& =Y_{k \delta}^{\varepsilon}+\frac{1}{\varepsilon} \int_{0}^{s} A Y_{\frac{r}{\varepsilon}}^{X_{k \delta}^{\varepsilon}, Y_{k \delta}^{\varepsilon}} d r+\frac{1}{\varepsilon} \int_{0}^{s} g\left(X_{k \delta}^{\varepsilon}, Y_{\frac{r}{\varepsilon}}^{X_{k \delta}^{\varepsilon}, Y_{k \delta}^{\varepsilon}}\right) d r+\frac{1}{\sqrt{\varepsilon}} \int_{0}^{s} d \overline{\bar{W}}^{Q_{2}}(r) .
\end{aligned}
$$

This, together with the uniqueness of the solution to the equation (3.22), implies that the distribution of $\left(X_{k \delta}^{\varepsilon}, \hat{Y}_{s+k \delta}^{\varepsilon}\right)$ coincides with the distribution of $\left(X_{k \delta}^{\varepsilon}, Y_{\underline{s}}^{X_{k \delta}^{\varepsilon}, Y_{k \delta}^{\varepsilon}}\right)$.

In order to estimate $\mathbb{E} \sup _{0 \leqslant t \leqslant T}\left\|J_{4}^{1}(t)\right\|^{2 p}$, we first give a control of $\mathbb{E} \sup _{0 \leqslant t \leqslant T}\left\|J_{4}^{1}(t)\right\|^{2}$ :

$$
\begin{aligned}
& \mathbb{E} \sup _{0 \leqslant t \leqslant T}\left\|J_{4}^{1}(t)\right\|^{2} \\
= & \mathbb{E} \sup _{0 \leqslant t \leqslant T}\left\|\sum_{k=0}^{n_{t}-1} e^{(t-(k+1) \delta) A} \int_{k \delta}^{(k+1) \delta} e^{((k+1) \delta-s) A}\left[f\left(X_{k \delta}^{\varepsilon}, \hat{Y}_{s}^{\varepsilon}\right)-\bar{f}\left(X_{k \delta}^{\varepsilon}\right)\right] d s\right\|^{2} \\
\leqslant & \mathbb{E} \sup _{0 \leqslant t \leqslant T}\left\{n_{t} \sum_{k=0}^{n_{t}-1}\left\|\int_{k \delta}^{(k+1) \delta} e^{((k+1) \delta-s) A}\left[f\left(X_{k \delta}^{\varepsilon}, \hat{Y}_{s}^{\varepsilon}\right)-\bar{f}\left(X_{k \delta}^{\varepsilon}\right)\right] d s\right\|^{2}\right\} \\
\leqslant & {\left[\frac{T}{\delta}\right] \sum_{k=0}^{\left[\frac{T}{\delta}\right]-1} \mathbb{E}\left\|\int_{k \delta}^{(k+1) \delta} e^{((k+1) \delta-s) A}\left[f\left(X_{k \delta}^{\varepsilon}, \hat{Y}_{s}^{\varepsilon}\right)-\bar{f}\left(X_{k \delta}^{\varepsilon}\right)\right] d s\right\|^{2} } \\
\leqslant & \frac{C_{T}}{\delta^{2}} \max _{0 \leqslant k \leqslant\left[\frac{T}{\delta}\right]-1} \mathbb{E}\left\|\int_{k \delta}^{(k+1) \delta} e^{((k+1) \delta-s) A}\left[f\left(X_{k \delta}^{\varepsilon}, \hat{Y}_{s}^{\varepsilon}\right)-\bar{f}\left(X_{k \delta}^{\varepsilon}\right)\right] d s\right\|^{2} \\
= & C_{T} \frac{\varepsilon^{2}}{\delta^{2}} \max _{0 \leqslant k \leqslant\left[\frac{T}{\delta}\right]-1} \mathbb{E}\left\|\int_{0}^{\frac{\delta}{\varepsilon}} e^{(\delta-s \varepsilon) A}\left[f\left(X_{k \delta}^{\varepsilon}, \hat{Y}_{s \varepsilon+k \delta}^{\varepsilon}\right)-\bar{f}\left(X_{k \delta}^{\varepsilon}\right)\right] d s\right\|^{2} \\
= & C_{T} \frac{\varepsilon^{2}}{\delta^{2}} \max _{0 \leqslant k \leqslant\left[\frac{T}{\delta}\right]-1} \int_{0}^{\frac{\delta}{\varepsilon}} \int_{\tau}^{\frac{\delta}{\varepsilon}} \Psi_{k}(s, \tau) d s d \tau,
\end{aligned}
$$

where

$$
\begin{aligned}
\Psi_{k}(s, \tau) & =\mathbb{E}\left\langle e^{(\delta-s \varepsilon) A}\left(f\left(X_{k \delta}^{\varepsilon}, \hat{Y}_{s \varepsilon+k \delta}^{\varepsilon}\right)-\bar{f}\left(X_{k \delta}^{\varepsilon}\right)\right), e^{(\delta-\tau \varepsilon) A}\left(f\left(X_{k \delta}^{\varepsilon}, \hat{Y}_{\tau \varepsilon+k \delta}^{\varepsilon}\right)-\bar{f}\left(X_{k \delta}^{\varepsilon}\right)\right)\right\rangle \\
& =\mathbb{E}\left\langle e^{(\delta-s \varepsilon) A}\left(f\left(X_{k \delta}^{\varepsilon}, Y_{s}^{X_{k \delta}^{\varepsilon}, Y_{k \delta}^{\varepsilon}}\right)-\bar{f}\left(X_{k \delta}^{\varepsilon}\right)\right), e^{(\delta-\tau \varepsilon) A}\left(f\left(X_{k \delta}^{\varepsilon}, Y_{\tau}^{X_{k \delta}^{\varepsilon}, Y_{k \delta}^{\varepsilon}}\right)-\bar{f}\left(X_{k \delta}^{\varepsilon}\right)\right)\right\rangle .
\end{aligned}
$$

Similar as the argument in [15, appendix A], using Lemma 3.1, one can verify that

$$
\Psi_{k}(s, \tau) \leqslant C \mathbb{E}\left(1+\left\|X_{k \delta}^{\varepsilon}\right\|^{2}+\left\|Y_{k \delta}^{\varepsilon}\right\|^{2}\right) e^{-\frac{1}{2}(s-\tau) \eta} \leqslant C_{T}\left(1+\|x\|^{2}+\|y\|^{2}\right) e^{-\frac{1}{2}(s-\tau) \eta} .
$$

Combining (3.36) and (3.37), we get that for any $\varepsilon \in(0,1)$

$$
\mathbb{E} \sup _{0 \leqslant t \leqslant T}\left\|J_{4}^{1}(t)\right\|^{2} \leqslant C_{T} \frac{\varepsilon}{\delta}\left(1+\|x\|^{2}+\|y\|^{2}\right) .
$$


By Lemmas 3.3 and 3.5, we have

$$
\begin{aligned}
\mathbb{E} \sup _{0 \leqslant t \leqslant T}\left\|J_{4}^{1}(t)\right\|^{2 p} & \leqslant \mathbb{E}\left(\int_{0}^{T}\left|f\left(X_{\left[\frac{s}{\delta}\right] \delta}^{\varepsilon}, \hat{Y}_{s}^{\varepsilon}\right)\right|+\bar{f}\left(X_{\left[\frac{s}{\delta}\right] \delta}^{\varepsilon}\right) d s\right)^{2 p} \\
& \leqslant C_{p, T}\left[1+\sup _{s \in[0, T]} \mathbb{E}\left(\left\|X_{s}^{\varepsilon}\right\|^{2 p}\right)+\sup _{s \in[0, T]} \mathbb{E}\left(\left\|\hat{Y}_{s}^{\varepsilon}\right\|^{2 p}\right)\right] \\
& \leqslant C_{p, T}\left(1+\|x\|^{2 p}+\|y\|^{2 p}\right) .
\end{aligned}
$$

This, together with (3.38), implies

$$
\begin{aligned}
\mathbb{E} \sup _{0 \leqslant t \leqslant T}\left\|J_{4}^{1}(t)\right\|^{2 p} & \leqslant\left(\mathbb{E} \sup _{0 \leqslant t \leqslant T}\left\|J_{4}^{1}(t)\right\|^{2(2 p-1)}\right)^{\frac{1}{2}}\left(\mathbb{E} \sup _{0 \leqslant t \leqslant T}\left\|J_{4}^{1}(t)\right\|^{2}\right)^{\frac{1}{2}} \\
& \leqslant C_{p, T}\left(1+\|x\|^{2 p}+\|y\|^{2 p}\right) \sqrt{\frac{\varepsilon}{\delta}}
\end{aligned}
$$

Consequently, combining (3.34), (3.35) and (3.40), we get

$$
\mathbb{E} \sup _{0 \leqslant t \leqslant T}\left\|J_{4}(t)\right\|^{2 p} \leqslant C_{p, T}\left(1+|x|_{\alpha}^{2 p}+\|y\|^{2 p}\right)\left(\delta^{p}+\delta^{2 p-1}+\sqrt{\frac{\varepsilon}{\delta}}\right) .
$$

According to the estimates (3.27)-(3.28), (3.31)-(3.33), (3.41), we obtain

$$
\begin{aligned}
\mathbb{E}\left(\sup _{0 \leqslant t \leqslant T \wedge \tau_{n}^{\varepsilon}}\left\|\hat{X}_{t}^{\varepsilon}-\bar{X}_{t}\right\|^{2 p}\right) \leqslant & C_{p, T}\left(1+|x|_{\alpha}^{6 p}+\|y\|^{6 p}\right)\left(\delta^{p}+\frac{\delta^{p+\frac{1}{2}}}{\sqrt{\varepsilon}}+\frac{\delta^{p+1}}{\varepsilon}+\delta^{2 p-1}+\sqrt{\frac{\varepsilon}{\delta}}\right) \\
& +C_{p, T} n^{2 p} \int_{0}^{T} \mathbb{E} \sup _{0 \leqslant r \leqslant s \wedge \tau_{n}^{\varepsilon}}\left\|\hat{X}_{r}^{\varepsilon}-\bar{X}_{r}\right\|^{2 p} d s .
\end{aligned}
$$

Using Gronwall's inequality, we get

$$
\begin{aligned}
& \mathbb{E}\left(\sup _{0 \leqslant t \leqslant T \wedge \tau_{n}^{\varepsilon}}\left\|\hat{X}_{t}^{\varepsilon}-\bar{X}_{t}\right\|^{2 p}\right) \\
\leqslant & C_{p, T}\left(1+|x|_{\alpha}^{6 p}+\|y\|^{6 p}\right)\left(\delta^{p}+\frac{\delta^{p+\frac{1}{2}}}{\sqrt{\varepsilon}}+\frac{\delta^{p+1}}{\varepsilon}+\delta^{2 p-1}+\sqrt{\frac{\varepsilon}{\delta}}\right) e^{C_{p, T} n^{2 p}},
\end{aligned}
$$

which implies

$$
\begin{aligned}
& \mathbb{E}\left(\sup _{0 \leqslant t \leqslant T}\left\|\hat{X}_{t}^{\varepsilon}-\bar{X}_{t}\right\|^{2 p} \cdot 1_{\left\{T \leqslant \tau_{n}^{\varepsilon}\right\}}\right) \\
\leqslant & C_{p, T}\left(1+|x|_{\alpha}^{2 p}+\|y\|^{2 p}\right)\left(\delta^{p}+\frac{\delta^{p+\frac{1}{2}}}{\sqrt{\varepsilon}}+\frac{\delta^{p+1}}{\varepsilon}+\delta^{2 p-1}+\sqrt{\frac{\varepsilon}{\delta}}\right) e^{C_{p, T} n^{2 p}} .
\end{aligned}
$$

Taking $n=\sqrt[2 p]{-\frac{1}{8 C_{p, T}} \log \varepsilon}, \delta=\varepsilon^{\frac{1}{2}}$, we get

$$
\mathbb{E}\left(\sup _{0 \leqslant t \leqslant T}\left\|\hat{X}_{t}^{\varepsilon}-\bar{X}_{t}\right\|^{2 p} \cdot 1_{\left\{T \leqslant \tau_{n}^{\varepsilon}\right\}}\right) \leqslant C_{p, T} \varepsilon^{\frac{1}{8}}\left(1+|x|_{\alpha}^{6 p}+\|y\|^{6 p}\right) .
$$


Note that, similarly to the proof of Lemma 3.3, one can check that uniformly in $\varepsilon \in(0,1)$, $\mathbb{E}\left(\sup _{0 \leqslant t \leqslant T}\left|\bar{X}_{t}^{\varepsilon}\right|_{1}\right) \leqslant C_{T}\left(1+|x|_{\alpha}\right)$. Combining this with (3.24), we deduce that

$$
\begin{aligned}
& \mathbb{E}\left(\sup _{0 \leqslant t \leqslant T}\left\|\hat{X}_{t}^{\varepsilon}-\bar{X}_{t}\right\|^{2 p} \cdot 1_{\left\{T>\tau_{n}^{\varepsilon}\right\}}\right) \leqslant\left(\mathbb{E} \sup _{0 \leqslant t \leqslant T}\left\|\hat{X}_{t}^{\varepsilon}-\bar{X}_{t}\right\|^{4 p}\right)^{\frac{1}{2}} \cdot\left[\mathbb{P}\left(T>\tau_{n}^{\varepsilon}\right)\right]^{\frac{1}{2}} \\
\leqslant & C_{p}\left(\mathbb{E}_{0 \leqslant t \leqslant T}\left\|\hat{X}_{t}^{\varepsilon}\right\|^{4 p}+\mathbb{E} \sup _{0 \leqslant t \leqslant T}\left\|\bar{X}_{t}\right\|^{4 p}\right)^{\frac{1}{2}} \frac{1}{\sqrt{n}}\left(\sup _{\varepsilon \in(0,1)} \mathbb{E} \sup _{0 \leqslant t \leqslant T}\left|\hat{X}_{t}^{\varepsilon}\right|_{1}+\mathbb{E} \sup _{0 \leqslant t \leqslant T}\left|\bar{X}_{t}\right|_{1}\right)^{\frac{1}{2}} \\
\leqslant & \frac{C_{p, T}}{\sqrt[4 p]{-\log \varepsilon}}\left(1+|x|_{\alpha}^{2 p+\frac{1}{2}}+\|y\|^{2 p+\frac{1}{2}}\right) .
\end{aligned}
$$

Putting together (3.42) and (3.43), we obtain

$$
\mathbb{E} \sup _{0 \leqslant t \leqslant T}\left\|\hat{X}_{t}^{\varepsilon}-\bar{X}_{t}\right\|^{2 p} \leqslant C_{p, T}\left(1+|x|_{\alpha}^{6 p}+\|y\|^{6 p}\right)\left(\frac{1}{-\log \varepsilon}\right)^{\frac{1}{4 p}} .
$$

The proof is complete.

3.4. Proof of Theorem 2.1. Taking $\delta=\varepsilon^{\frac{1}{2}}$, Lemma 3.7 implies

$$
\mathbb{E} \sup _{0 \leqslant t \leqslant T}\left\|X_{t}^{\varepsilon}-\hat{X}_{t}^{\varepsilon}\right\|^{2 p} \leqslant C_{p, T}\left(1+|x|_{\alpha}^{2 p}+\|y\|^{2 p}\right) \varepsilon^{\frac{p}{2}-\frac{1}{2}} .
$$

Combining this with Lemma 3.10, we obtain

$$
\begin{aligned}
\mathbb{E} \sup _{0 \leqslant t \leqslant T}\left\|X_{t}^{\varepsilon}-\bar{X}_{t}\right\|^{2 p} & \leqslant \mathbb{E} \sup _{0 \leqslant t \leqslant T}\left\|X_{t}^{\varepsilon}-\hat{X}_{t}\right\|^{2 p}+\mathbb{E} \sup _{0 \leqslant t \leqslant T}\left\|\hat{X}_{t}^{\varepsilon}-\bar{X}_{t}\right\|^{2 p} \\
& \leqslant C_{p, T}\left(1+|x|_{\alpha}^{6 p}+\|y\|^{6 p}\right)\left(\frac{1}{-\log \varepsilon}\right)^{\frac{1}{4 p}} \longrightarrow 0 \quad(\varepsilon \rightarrow 0),
\end{aligned}
$$

which concludes the proof of Theorem 2.1.

\section{Proofs of Theorems 2.2 And 2.3}

This section is devoted to proving Theorems 2.2 and 2.3. Following the procedure inspired by [3], the proofs are based on the Galerkin approximation and the asymptotic expansion with respect to $\varepsilon$ of the solution to the Kolmogorov equation corresponding to (2.2) with $Q_{1}=0$. Since the proofs are tediously long and technical, we first give a brief summary of the main ideas and steps in the proofs of Theorems 2.2 and 2.3.

Step 1. Due to the unboundedness of operator $\Delta$, we use the Galerkin approximation to reduce the infinite dimensional problem to a finite dimensional one as follows.

Let $H_{N}=\operatorname{span}\left\{e_{k} ; 1 \leqslant k \leqslant N\right\}$. Denote by $P_{N}$ the orthogonal projection of $L^{2}$ onto $H_{N}$. Set $f_{N}(x, y)=P_{N}(f(x, y)), g_{N}(x, y)=P_{N}(g(x, y)), B_{N}(x)=P_{N}(B(x)), W_{N}^{Q_{2}}(t)=$ $P_{N} W^{Q_{2}}(t)$ for $x, y \in H_{N}$. The following equation is the finite dimensional projection of the system (2.2) with $Q_{1}=0$ :

$$
\begin{cases}d X_{N}^{\varepsilon}(t)=\left[A X_{N}^{\varepsilon}(t)+B_{N}\left(X_{N}^{\varepsilon}(t)\right)+f_{N}\left(X_{N}^{\varepsilon}(t), Y_{N}^{\varepsilon}(t)\right)\right] d t, & X_{N}^{\varepsilon}(0)=P_{N} x \\ d Y_{N}^{\varepsilon}(t)=\frac{1}{\varepsilon}\left[A Y_{N}^{\varepsilon}(t)+g_{N}\left(X_{N}^{\varepsilon}(t), Y_{N}^{\varepsilon}(t)\right)\right] d t+\frac{1}{\sqrt{\varepsilon}} d W_{N}^{Q_{2}}(t), & Y_{N}^{\varepsilon}(0)=P_{N} y\end{cases}
$$

Similarly, we consider the finite dimensional projection of the equation (1.3) with $Q_{1}=0$ :

$$
\left\{\begin{array}{l}
d \bar{X}_{N}(t)=\left[A \bar{X}_{N}(t)+B_{N}\left(\bar{X}_{N}(t)\right)+\bar{f}_{N}\left(\bar{X}_{N}(t)\right)\right] d t \\
\bar{X}_{N}(0)=P_{N} x
\end{array}\right.
$$


where $\bar{f}_{N}(x)=\int_{H_{N}} P_{N} f(x, y) \mu_{N}^{x}(d y)$, and $\mu_{N}^{x}(d y)$ is the unique invariant measure for

$$
d Y_{N}(t)=\left[A Y_{N}(t)+g_{N}\left(x, Y_{N}(t)\right)\right] d t+d W_{N}^{Q_{2}}(t)
$$

For the test function $\phi \in C_{b}^{2}\left(L^{2}\right)$, we have

$$
\begin{aligned}
& \mathbb{E}\left[\phi\left(X^{\varepsilon}(t)\right)\right]-\phi(\bar{X}(t)) \\
= & \left\{\mathbb{E}\left[\phi\left(X^{\varepsilon}(t)\right)\right]-\mathbb{E}\left[\phi\left(X_{N}^{\varepsilon}(t)\right)\right]\right\}+\left\{\mathbb{E}\left[\phi\left(X_{N}^{\varepsilon}(t)\right)\right]-\phi\left(\bar{X}_{N}(t)\right)\right\}+\left\{\phi\left(\bar{X}_{N}(t)\right)-\phi(\bar{X}(t))\right\} .
\end{aligned}
$$

It is not difficult to show that the first term and the third term in (4.3) converge to 0, as $N \rightarrow \infty$. Therefore, in order to establish Theorems 2.2 and 2.3, it remains to show that the second term in (4.3) converges to 0 as $N \rightarrow \infty$. We will give the main idea in the next step.

Step 2. Inspired by [3], we construct an asymptotic expansion of $\mathbb{E}\left[\phi\left(X_{N}^{\varepsilon}(t)\right)\right]$. Roughly speaking, it has an expansion with respect to the small parameter $\varepsilon$ :

$$
\mathbb{E}\left[\phi\left(X_{N}^{\varepsilon}(t)\right)\right]=\phi\left(\bar{X}_{N}(t)\right)+\varepsilon u_{1}+v^{\varepsilon} .
$$

In order to control the second term in (4.3), the main task is to analyze $u_{1}$ and $v^{\varepsilon}$. Almost all the work in this section is to deal with this step.

This section is organized as follows. Subsections 4.1 and 4.2 are to establish some properties of $\bar{X}_{N}$ and $\left(X_{N}^{\varepsilon}, Y_{N}^{\varepsilon}\right)$ respectively. The asymptotic expansion of $\mathbb{E}\left[\phi\left(X_{N}^{\varepsilon}(t)\right)\right]$ will be given in Subsection 4.3. Based on some results obtained in subsections 4.1 and 4.2, subsection 4.4 is to study properties of $u_{1}$ and $v^{\varepsilon}$. Finally, we prove Theorems 2.2 and 2.3 in subsections 4.5 and 4.6 , respectively.

4.1. Properties of $\bar{X}_{N}$. This subsection is to establish some properties of $\bar{X}_{N}$. For simplicity, we omit the index $N$.

Lemma 4.1. Assume the conditions A1, A2 and A4 hold.

(1) For any $x \in L^{2}, T>0$, there exists a constant $C>0$ such that

$$
\sup _{0 \leqslant t \leqslant T}\left\|\bar{X}_{t}\right\| \leqslant C(1+\|x\|) .
$$

(2) Furthermore, for any $x \in H^{\theta}$ with $\theta \in(0,1), \gamma \in\left(1, \frac{3}{2}\right), t \in(0, T]$, there exist $k \in \mathbb{N}$ and a constant $C=C_{\gamma, \theta, T}>0$ such that

$$
\left|\bar{X}_{t}\right|_{\gamma} \leqslant C\left(|x|_{\theta}+1\right) t^{-\frac{\gamma-\theta}{2}} e^{C\|x\|^{k}} .
$$

Proof. Multiplying both sides of the equation (4.2) by $2 \bar{X}_{t}$ and integrating with respect to $\xi$, we get

$$
\frac{d}{d t}\left\|\bar{X}_{t}\right\|^{2}=2\left\langle A \bar{X}_{t}, \bar{X}_{t}\right\rangle+2\left\langle\bar{f}\left(\bar{X}_{t}\right), \bar{X}_{t}\right\rangle \leqslant C\left(1+\left\|\bar{X}_{t}\right\|^{2}\right)
$$

which implies (4.4) by applying Gronwall's inequality.

To prove (4.5), note that

$$
\bar{X}_{t}=e^{t A} x+\int_{0}^{t} e^{(t-s) A} B\left(\bar{X}_{s}\right) d s+\int_{0}^{t} e^{(t-s) A} \bar{f}\left(\bar{X}_{s}\right) d s .
$$

For the first term, we have

$$
\left|e^{t A} x\right|_{\gamma} \leqslant C t^{-\frac{\gamma-\theta}{2}}|x|_{\theta}
$$


For the second term, it follows from (2.1) and Lemma 5.2 that

$$
\begin{aligned}
\left|\int_{0}^{t} e^{(t-s) A} B\left(\bar{X}_{s}\right) d s\right|_{\gamma} & \leqslant C \int_{0}^{t}\left[1+(t-s)^{-\frac{1+2 \gamma}{4}}\right]\left|B\left(\bar{X}_{s}\right)\right|_{-\frac{1}{2}} d s \\
& \leqslant C \int_{0}^{t}(t-s)^{-\frac{1+2 \gamma}{4}}\left\|\bar{X}_{s}\right\|\left|\bar{X}_{s}\right|_{\gamma} d s \\
& \leqslant C \int_{0}^{t}(t-s)^{-\frac{1+2 \gamma}{4}}(1+\|x\|)\left|\bar{X}_{s}\right|_{\gamma} d s .
\end{aligned}
$$

For the last term, using (2.1) and (4.4), we get

$$
\left|\int_{0}^{t} e^{(t-s) A} \bar{f}\left(\bar{X}_{s}\right) d s\right|_{\gamma} \leqslant C \int_{0}^{t}\left[1+(t-s)^{-\frac{\gamma}{2}}\right]\left(1+\left\|\bar{X}_{s}\right\|\right) d s \leqslant C(1+\|x\|) .
$$

Consequently, combining (4.6)-(4.8), we conclude the proof of (4.5) by using Lemma 5.5.

Note that from Lemma 4.1, using the interpolation inequality, we get that for any $\gamma \in(0,1]$, $\theta \in(0,1), \delta \in\left(0, \frac{1}{2}\right), t \in(0, T]$, there exist $k \in \mathbb{N}$ and a constant $C=C_{\theta, \delta, T}>0$ such that

$$
\left|\bar{X}_{t}\right|_{\gamma} \leqslant C\left\|\bar{X}_{t}\right\|^{\frac{1+\delta-\gamma}{1+\delta}}\left|\bar{X}_{t}\right|_{1+\delta}^{\frac{\gamma}{1+\delta}} \leqslant C t^{-\frac{1+\delta-\theta}{2} \frac{\gamma}{1+\delta}}\left(|x|_{\theta}+1\right) e^{C\|x\|^{k}}
$$

Lemma 4.2. Under the conditions $\boldsymbol{A} 1, \boldsymbol{A} \mathscr{2}$ and $\boldsymbol{A} 4$, for any $\theta \in(0,1), \alpha \in\left(0, \frac{1}{2}\right), x \in H^{\theta}$, $0<s<t \leqslant T$, there exist $k \in \mathbb{N}$ and a constant $C=C_{\theta, \alpha, T}>0$ such that

$$
|\bar{X}(t, x)-\bar{X}(s, x)|_{1} \leqslant C(t-s)^{\frac{\alpha}{2}} s^{-\frac{1+\alpha-\theta}{2}}\left(|x|_{\theta}+1\right) e^{C\|x\|^{k}} .
$$

Proof. In view of (4.2), we write

$$
\begin{aligned}
& \bar{X}(t, x)-\bar{X}(s, x) \\
= & \left(e^{A(t-s)}-I\right) \bar{X}(s, x)+\int_{s}^{t} e^{(t-r) A} B(\bar{X}(r, x)) d r+\int_{s}^{t} e^{(t-r) A} \bar{f}(\bar{X}(r, x)) d r .
\end{aligned}
$$

For the first term, using the property $\left\|\left(e^{t A}-I\right) x\right\| \leqslant C t^{\frac{\alpha}{2}}|x|_{\alpha}$ and Lemma 4.1, we get that there exists $k \in \mathbb{N}$ such that

$$
\begin{aligned}
\left|\left(e^{A(t-s)}-I\right) \bar{X}(s, x)\right|_{1} & \leqslant C(t-s)^{\frac{\alpha}{2}}|\bar{X}(s, x)|_{1+\alpha} \\
& \leqslant C(t-s)^{\frac{\alpha}{2}} s^{-\frac{1+\alpha-\theta}{2}}\left(|x|_{\theta}+1\right) e^{C\|x\|^{k}} .
\end{aligned}
$$

For the second term, according to Lemma 4.1, there exists some $k \in \mathbb{N}$ such that

$$
\begin{aligned}
\left|\int_{s}^{t} e^{(t-r) A} B(\bar{X}(r, x)) d r\right|_{1} & \leqslant C \int_{s}^{t}\left[1+(t-r)^{-\frac{3}{4}}\right]|B(\bar{X}(r, x))|_{-\frac{1}{2}} d r \\
& \leqslant C \int_{s}^{t}\left[1+(t-r)^{-\frac{3}{4}}\right]\|\bar{X}(r, x)\||\bar{X}(r, x)|_{1+\alpha} d r \\
& \leqslant C \int_{s}^{t}\left[1+(t-r)^{-\frac{3}{4}}\right] r^{-\frac{1+\alpha-\theta}{2}}\left(|x|_{\theta}+1\right) e^{C\|x\|^{k}} d r \\
& \leqslant C(t-s)^{\frac{1}{4}} s^{-\frac{1+\alpha-\theta}{2}}\left(|x|_{\theta}+1\right) e^{C\|x\|^{k}} .
\end{aligned}
$$


For the third term, using Lemma 4.1 again, we obtain

$$
\begin{aligned}
\left|\int_{s}^{t} e^{(t-r) A} \bar{f}(\bar{X}(r, x)) d r\right|_{1} & \leqslant C \int_{s}^{t}\left[1+(t-r)^{-\frac{1}{2}}\right]\|\bar{f}(\bar{X}(r, x))\| d r \\
& \leqslant C \int_{s}^{t}\left[1+(t-r)^{-\frac{1}{2}}\right](1+\|\bar{X}(r, x)\|) d r \\
& \leqslant C(t-s)^{\frac{1}{2}}(1+\|x\|) .
\end{aligned}
$$

The result follows by combining (4.10)-(4.13).

Lemma 4.3. Under the conditions $\boldsymbol{A 1}, \boldsymbol{A} \mathscr{2}$ and $\boldsymbol{A 4}$, for any $x \in H^{\theta}$ with $\theta \in(0,1)$, $0 \leqslant t \leqslant T$, there exist $k \in \mathbb{N}$ and a constant $C=C_{\theta, T}>0$ such that

$$
\left\|\frac{d}{d t} \bar{X}(t, x)\right\| \leqslant C t^{-1+\frac{\theta}{2}}\left(|x|_{\theta}^{2}+1\right) e^{C\|x\|^{k}}
$$

Proof. Recall that

$$
\frac{d}{d t} \bar{X}(t, x)=A \bar{X}(t, x)+B(\bar{X}(t, x))+\bar{f}(\bar{X}(t, x)) .
$$

Taking $\delta=\frac{1}{4}$ in (4.9), we get $\|B(\bar{X}(t, x))\| \leqslant C|\bar{X}(t, x)|_{1}^{2} \leqslant C t^{-1+\frac{4 \theta}{5}}\left(|x|_{\theta}^{2}+1\right) e^{C\|x\|^{k}}$. It is easy to see that $\|\bar{f}(\bar{X}(t, x))\| \leqslant C(1+\|x\|)$. Hence, to prove Lemma 4.3, it remains to control the first term in (4.14). From (4.14), we write

$$
\begin{aligned}
\bar{X}(t, x)= & e^{t A} x+\int_{0}^{t} e^{(t-s) A} B(\bar{X}(t, x)) d s+\int_{0}^{t} e^{(t-s) A}[B(\bar{X}(s, x))-B(\bar{X}(t, x))] d s \\
& +\int_{0}^{t} e^{(t-s) A} \bar{f}(\bar{X}(t, x)) d s+\int_{0}^{t} e^{(t-s) A}[\bar{f}(\bar{X}(s, x))-\bar{f}(\bar{X}(t, x))] d s \\
:= & I_{1}+I_{2}+I_{3}+I_{4}+I_{5} .
\end{aligned}
$$

For $I_{1}$, using (2.1), we have

$$
\left\|A I_{1}\right\| \leqslant C t^{-1+\frac{\theta}{2}}|x|_{\theta}
$$

For $I_{2}$, we deduce from Corollary 5.3 and (4.9) that

$$
\begin{aligned}
\left\|A I_{2}\right\| & =\left\|\left(e^{t A}-I\right) B(\bar{X}(t, x))\right\| \leqslant 2\|B(\bar{X}(t, x))\| \\
& \leqslant 2|\bar{X}(t, x)|_{1}^{2} \leqslant C t^{-1+\frac{4 \theta}{5}}\left(|x|_{\theta}^{2}+1\right) e^{C\|x\|^{k}} .
\end{aligned}
$$

For $I_{3}$, according to Lemma 4.2 and (4.9), we get

$$
\begin{aligned}
\left\|A I_{3}\right\| & \leqslant C \int_{0}^{t} \frac{1}{t-s}\|B(\bar{X}(t, x))-B(\bar{X}(s, x))\| d s \\
& \leqslant C \int_{0}^{t} \frac{1}{t-s}|\bar{X}(t, x)-\bar{X}(s, x)|_{1}\left(|\bar{X}(t, x)|_{1}+|\bar{X}(s, x)|_{1}\right) d s \\
& \leqslant C \int_{0}^{t} \frac{1}{t-s}(t-s)^{\frac{\alpha}{2}} s^{-\frac{1+\alpha-\theta}{2}}\left(t^{-\frac{1}{2}+\frac{2 \theta}{5}}+s^{-\frac{1}{2}+\frac{2 \theta}{5}}\right)\left(|x|_{\theta}^{2}+1\right) e^{C\|x\|^{k}} d s \\
& \leqslant C t^{-1+\frac{9 \theta}{10}}\left(|x|_{\theta}^{2}+1\right) e^{C\|x\|^{k}} .
\end{aligned}
$$

For $I_{4}$, it follows from Lemma 4.1 that

$$
\left\|A I_{4}\right\|=\left\|\left(e^{t A}-I\right) \bar{f}(\bar{X}(t, x))\right\| \leqslant C(1+\|\bar{X}(t, x)\|) \leqslant C(1+\|x\|) .
$$


For $I_{5}$, using Lemma 4.2 gives

$$
\left\|A I_{5}\right\| \leqslant C \int_{0}^{t} \frac{1}{t-s}\|\bar{X}(t, x)-\bar{X}(s, x)\| d s \leqslant C t^{-\frac{1-\theta}{2}}\left(|x|_{\theta}+1\right) e^{C\|x\|^{k}} .
$$

The conclusion follows by the above estimates.

Denote by $\eta^{h}(t, x)$ the derivative of $\bar{X}(t, x)$ with respect to $x$ in the direction $h . \eta^{h}(t, x)$ satisfies the following equation

$$
\left\{\begin{array}{l}
\frac{d \eta^{h}(t, x)}{d t}=A \eta^{h}(t, x)+D \bar{f}(\bar{X}(t, x)) \cdot \eta^{h}(t, x)+D_{\xi}\left[\bar{X}(t, x) \eta^{h}(t, x)\right] \\
\eta^{h}(0, x)=h .
\end{array}\right.
$$

The following three Lemmas give some bounds for $\eta^{h}(t, x)$.

Lemma 4.4. Assume the conditions A1, A2 and A4 hold.

(1) For any $t \in(0, T], h \in L^{2}$, there exists a constant $C>0$ such that

$$
\left\|\eta^{h}(t, x)\right\|^{2}+\int_{0}^{t}\left|\eta^{h}(s, x)\right|_{1}^{2} d s \leqslant C e^{C\|x\|^{5}}\|h\|^{2} .
$$

(2) For any $x \in H^{\theta}$ with $\theta \in(0,1), h \in L^{2}, \gamma \in\left(1, \frac{3}{2}\right), t \in(0, T]$, there exist $k \in \mathbb{N}$ and $a$ constant $C=C_{\gamma, \theta, T}$ such that

$$
\left|\eta^{h}(t, x)\right|_{\gamma} \leqslant C t^{-\frac{\gamma}{2}}\left(|x|_{\theta}+1\right) e^{C\|x\|^{k}}\|h\| .
$$

Proof. Multiplying both sides of the equation (4.15) by $\eta^{h}(t, x)$ and integrating with respect to $\xi$, we obtain

$$
\begin{aligned}
\frac{1}{2} \frac{d}{d t}\left\|\eta^{h}(t, x)\right\|^{2}+\left|\eta^{h}(t, x)\right|_{1}^{2}= & \int_{0}^{1}\left[D \bar{f}(\bar{X}(t, x)) \eta^{h}(t, x)\right] \eta^{h}(t, x) d \xi \\
& +\int_{0}^{1} D_{\xi}\left[\bar{X}(t, x) \eta^{h}(t, x)\right] \eta^{h}(t, x) d \xi \\
\leqslant & C\left\|\eta^{h}(t, x)\right\|^{2}-\int_{0}^{1} \bar{X}(t, x) \eta^{h}(t, x) D_{\xi} \eta^{h}(t, x) d \xi \\
= & C\left\|\eta^{h}(t, x)\right\|^{2}-b\left(\bar{X}(t, x), \eta^{h}(t, x), \eta^{h}(t, x)\right) .
\end{aligned}
$$

According to Lemma 5.2 and the interpolation inequality, it follows that

$$
\begin{aligned}
\frac{1}{2} \frac{d}{d t}\left\|\eta^{h}(t, x)\right\|^{2}+\left|\eta^{h}(t, x)\right|_{1}^{2} & \leqslant C\left\|\eta^{h}(t, x)\right\|^{2}+\|\bar{X}(t, x)\|\left|\eta^{h}(t, x)\right|_{1}\left|\eta^{h}(t, x)\right|_{\frac{3}{5}} \\
& \leqslant C\left\|\eta^{h}(t, x)\right\|^{2}+\|\bar{X}(t, x)\|\left|\eta^{h}(t, x)\right|_{1}^{\frac{8}{5}}\left\|\eta^{h}(t, x)\right\|^{\frac{2}{5}} \\
& \leqslant C\left\|\eta^{h}(t, x)\right\|^{2}+\frac{1}{2}\left|\eta^{h}(t, x)\right|_{1}^{2}+C\|\bar{X}(t, x)\|^{5}\left\|\eta^{h}(t, x)\right\|^{2} .
\end{aligned}
$$

This implies

$$
\left\|\eta^{h}(t, x)\right\|^{2}+\int_{0}^{t}\left|\eta^{h}(s, x)\right|_{1}^{2} d s \leqslant\|h\|^{2}+C \int_{0}^{t}\left(1+\|\bar{X}(s, x)\|^{5}\right)\left\|\eta^{h}(s, x)\right\|^{2} d s .
$$

Then, (4.16) follows by using Gronwall's inequality and Lemma 4.1.

To show (4.17), notice that

$$
\eta^{h}(t, x)=e^{t A} h+\int_{0}^{t} e^{(t-s) A} D \bar{f}(\bar{X}(s, x)) \cdot \eta^{h}(s, x) d s+\int_{0}^{t} e^{(t-s) A} D_{\xi}\left[\bar{X}(s, x) \eta^{h}(s, x)\right] d s .
$$


Using (4.16), Lemmas 5.2 and 4.1, we obtain that for $\gamma \in\left(1, \frac{3}{2}\right)$,

$$
\begin{aligned}
\left|\eta^{h}(t, x)\right|_{\gamma} \leqslant & C t^{-\frac{\gamma}{2}}\|h\|+C \int_{0}^{t}(t-s)^{-\frac{\gamma}{2}}\left|D \bar{f}(\bar{X}(s, x)) \cdot \eta^{h}(s, x)\right| d s \\
& +C \int_{0}^{t}(t-s)^{-\frac{2 \gamma+1}{4}}\left|D_{\xi}\left[\bar{X}(s, x) \eta^{h}(s, x)\right]\right|_{-\frac{1}{2}} d s \\
\leqslant & C t^{-\frac{\gamma}{2}}\|h\|+C \int_{0}^{t}(t-s)^{-\frac{\gamma}{2}}\left\|\eta^{h}(s, x)\right\| d s \\
& +C \int_{0}^{t}(t-s)^{-\frac{2 \gamma+1}{4}}\left|B\left(\bar{X}(s, x), \eta^{h}(s, x)\right)+B\left(\eta^{h}(s, x), \bar{X}(s, x)\right)\right|_{-\frac{1}{2}} d s \\
\leqslant & C t^{-\frac{\gamma}{2}}\|h\|+C e^{C|x|^{5}}\|h\| \\
& +C \int_{0}^{t}(t-s)^{-\frac{2 \gamma+1}{4}}\left[\left\|\eta^{h}(s, x)\right\||\bar{X}(s, x)|_{\gamma}+\|\bar{X}(s, x)\|\left|\eta^{h}(s, x)\right|_{\gamma}\right] d s \\
\leqslant & C t^{-\frac{\gamma}{2}}\left(|x|_{\theta}+1\right) e^{C\|x\|^{k}}\|h\|+\int_{0}^{t}(t-s)^{-\frac{2 \gamma+1}{4}}\left|\eta^{h}(s, x)\right|_{\gamma}(1+\|x\|) d s .
\end{aligned}
$$

Consequently, by Lemma 5.5, we get (4.17). The proof is complete.

Note that from Lemma 4.4, using the interpolation inequality, we deduce that for any $\gamma \in(0,1], \theta \in(0,1), t \in(0, T]$, there exist $k \in \mathbb{N}$ and a constant $C=C_{\theta, T}>0$ such that

$$
\left|\eta^{h}(t, x)\right|_{\gamma} \leqslant C t^{-\frac{\gamma}{2}}\left(|x|_{\theta}+1\right) e^{C\|x\|^{k}}\|h\| .
$$

Lemma 4.5. Under the conditions $\boldsymbol{A 1}, \boldsymbol{A} \mathscr{2}$ and $\boldsymbol{A 4}$, for any $x \in H^{\theta}$ with $\theta \in(0,1)$, $\alpha \in\left(0, \frac{1}{2}\right), 0<s<t \leqslant T$, there exist $k \in \mathbb{N}$ and a constant $C=C_{\alpha, \theta, T}$ such that

$$
\left|\eta^{h}(t, x)-\eta^{h}(s, x)\right|_{1} \leqslant C(t-s)^{\frac{\alpha}{2}} s^{-\frac{1+\alpha}{2}}\left(1+|x|_{\theta}\right) e^{C\|x\|^{k}}\|h\| .
$$

Proof. From (4.15), we write

$$
\begin{aligned}
\eta^{h}(t, x)-\eta^{h}(s, x)= & \left(e^{(t-s) A}-I\right) \eta^{h}(s, x)+\int_{s}^{t} e^{(t-r) A} D \bar{f}(\bar{X}(r, x)) \cdot \eta^{h}(r, x) d r \\
& +\int_{s}^{t} e^{(t-r) A} D_{\xi}\left[\bar{X}(r, x) \eta^{h}(r, x)\right] d r \\
= & I_{1}+I_{2}+I_{3} .
\end{aligned}
$$

For $I_{1}$, using (2.1) and (4.17), we have

$$
\left|I_{1}\right|_{1} \leqslant C(t-s)^{\frac{\alpha}{2}}\left|\eta^{h}(s, x)\right|_{1+\alpha} \leqslant C(t-s)^{\frac{\alpha}{2}} s^{-\frac{1+\alpha}{2}}\left(1+|x|_{\theta}\right) e^{C\|x\|^{k}}\|h\| .
$$

For $I_{2}$, using (4.16), we get

$$
\left|I_{2}\right|_{1} \leqslant \int_{s}^{t}(t-r)^{-\frac{1}{2}}\left\|D \bar{f}(\bar{X}(r, x)) \cdot \eta^{h}(r, x)\right\| d r \leqslant C(t-s)^{\frac{1}{2}} e^{C\|x\|^{5}}\|h\| .
$$

For $I_{3}$, using (2.1), Lemmas 4.1 and 4.4, we obtain

$$
\begin{aligned}
\left|I_{3}\right|_{1} & \leqslant C \int_{s}^{t}\left[1+(t-r)^{-\frac{3}{4}}\right]\left|D_{\xi}\left[\bar{X}(r, x) \eta^{h}(r, x)\right]\right|_{-\frac{1}{2}} d r \\
& \leqslant C \int_{s}^{t}\left[1+(t-r)^{-\frac{3}{4}}\right]\left|B\left(\bar{X}(r, x), \eta^{h}(r, x)\right)+B\left(\eta^{h}(r, x), \bar{X}(r, x)\right)\right|_{-\frac{1}{2}} d r \\
& \leqslant C \int_{s}^{t}\left[1+(t-r)^{-\frac{3}{4}}\right]\left(\|\bar{X}(r, x)\|\left|\eta^{h}(r, x)\right|_{1+\alpha}+|\bar{X}(r, x)|_{1+\alpha}\left\|\eta^{h}(r, x)\right\|\right) d r \\
& \leqslant C(t-s)^{\frac{1}{4}} s^{-\frac{1+\alpha}{2}}\left(1+|x|_{\theta}\right) e^{C\|x\|^{k}}\|h\| .
\end{aligned}
$$


We conclude the proof by combining (4.20)-(4.23).

Lemma 4.6. Under the conditions $\boldsymbol{A 1}, \boldsymbol{A} \mathscr{2}$ and $\boldsymbol{A} 4$, for any $x \in H^{\theta}$ with $\theta \in(0,1), h \in L^{2}$, $0<s<t \leqslant T$, there exist $k \in \mathbb{N}$ and a constant $C=C_{\theta, T}$ such that

$$
\left\|\frac{d}{d t} \eta^{h}(t, x)\right\| \leqslant C t^{-1}\left(|x|_{\theta}^{2}+1\right) e^{C\|x\|^{k}}\|h\| .
$$

Proof. We first control the first term in (4.15). Notice that

$$
\begin{aligned}
\eta^{h}(t, x)= & e^{t A} h+\int_{0}^{t} e^{(t-s) A} D \bar{f}(\bar{X}(t, x)) \cdot \eta^{h}(t, x) d s \\
& +\int_{0}^{t} e^{(t-s) A}\left[D \bar{f}(\bar{X}(s, x)) \cdot \eta^{h}(s, x)-D \bar{f}(\bar{X}(t, x)) \cdot \eta^{h}(t, x)\right] d s \\
& +\int_{0}^{t} e^{(t-s) A} D_{\xi}\left[\bar{X}(t, x) \eta^{h}(t, x)\right] d s \\
& +\int_{0}^{t} e^{(t-s) A}\left\{D_{\xi}\left[\bar{X}(s, x) \eta^{h}(s, x)\right]-D_{\xi}\left[\bar{X}(t, x) \eta^{h}(t, x)\right]\right\} d s \\
= & I_{1}+I_{2}+I_{3}+I_{4}+I_{5} .
\end{aligned}
$$

For $I_{1}$, we have

$$
\left\|A I_{1}\right\| \leqslant C t^{-1}\|h\|
$$

For $I_{2}$, it follows from condition $\mathrm{A} 4$ that

$$
\left\|A I_{2}\right\|=\left\|\left(e^{t A}-I\right) D \bar{f}(\bar{X}(t, x)) \cdot \eta^{h}(t, x)\right\| \leqslant 2\left\|D \bar{f}(\bar{X}(t, x)) \cdot \eta^{h}(t, x)\right\| \leqslant C e^{C\|x\|^{5}}\|h\| .
$$

For $I_{3}$, according to Lemmas 4.2 and 4.5, we obtain

$$
\begin{aligned}
\left\|A I_{3}\right\| \leqslant & C \int_{0}^{t} \frac{1}{t-s}\left\|D \bar{f}(\bar{X}(t, x)) \cdot \eta^{h}(t, x)-D \bar{f}(\bar{X}(s, x)) \cdot \eta^{h}(s, x)\right\| d s \\
\leqslant & C \int_{0}^{t} \frac{1}{t-s}\left\|[D \bar{f}(\bar{X}(t, x))-D \bar{f}(\bar{X}(s, x))] \cdot \eta^{h}(t, x)\right\| d s \\
& +C \int_{0}^{t} \frac{1}{t-s}\left\|D \bar{f}(\bar{X}(s, x)) \cdot\left(\eta^{h}(t, x)-\eta^{h}(s, x)\right)\right\| d s \\
\leqslant & C \int_{0}^{t} \frac{1}{t-s}\|\bar{X}(t, x)-\bar{X}(s, x)\| e^{C\|x\|^{5}}\|h\| d s+C \int_{0}^{t} \frac{1}{t-s}\left\|\eta^{h}(t, x)-\eta^{h}(s, x)\right\| d s \\
\leqslant & C t^{-\frac{1}{2}}\left(1+|x|_{\theta}\right) e^{C\|x\|^{k}}\|h\| .
\end{aligned}
$$

For $I_{4}$, we deduce from Lemma 5.2, (4.9) and (4.19) that

$$
\begin{aligned}
\left\|A I_{4}\right\| & =\left\|\left(e^{t A}-I\right) D_{\xi}\left[\bar{X}(t, x) \eta^{h}(t, x)\right]\right\| \\
& \leqslant 2\left\|B\left(\bar{X}(t, x), \eta^{h}(t, x)\right)+B\left(\eta^{h}(t, x), \bar{X}(t, x)\right)\right\| \\
& \leqslant C|\bar{X}(t, x)|_{1}\left|\eta^{h}(t, x)\right|_{1} \leqslant C t^{-1+\frac{2 \theta}{5}}\left(|x|_{\theta}^{2}+1\right) e^{C\|x\|^{k}}\|h\| .
\end{aligned}
$$


For $I_{5}$, using Lemmas 4.2 and 4.5, (4.9) and (4.19), we get

$$
\begin{aligned}
\left\|A I_{5}\right\| \leqslant & C \int_{0}^{t} \frac{1}{t-s} \| B\left(\bar{X}(s, x), \eta^{h}(t, x)-\eta^{h}(s, x)\right)+B\left(\eta^{h}(t, x)-\eta^{h}(s, x), \bar{X}(s, x)\right) \\
& +B\left(\bar{X}(t, x)-\bar{X}(s, x), \eta^{h}(t, x)\right)+B\left(\eta^{h}(t, x), \bar{X}(t, x)-\bar{X}(s, x)\right) \| d s \\
\leqslant & C \int_{0}^{t} \frac{1}{t-s}\left(|\bar{X}(s, x)|_{1}\left|\eta^{h}(t, x)-\eta^{h}(s, x)\right|_{1}+\left|\eta^{h}(t, x)\right|_{1}|\bar{X}(t, x)-\bar{X}(s, x)|_{1}\right) d s \\
\leqslant & C t^{-1+\frac{2 \theta}{5}}\left(|x|_{\theta}^{2}+1\right) e^{C\|x\|^{k}}\|h\| .
\end{aligned}
$$

Putting (4.24)-(4.28) together, we get

$$
\left\|A \eta^{h}(t, x)\right\| \leqslant C t^{-1}\left(|x|_{\theta}^{2}+1\right) e^{C\|x\|^{k}}\|h\| .
$$

For the second term in (4.15), we have

$$
\left\|D \bar{f}(\bar{X}(t, x)) \cdot \eta^{h}(t, x)\right\| \leqslant C e^{C\|x\|^{5}}\|h\| .
$$

For the third term in (4.15), it follows by (4.27) that

$$
\left\|D_{\xi}\left[\bar{X}(t, x) \eta^{h}(t, x)\right]\right\| \leqslant C t^{-1+\frac{2 \theta}{5}}\left(|x|_{\theta}^{2}+1\right) e^{C\|x\|^{k}}\|h\| .
$$

The result follows by combining (4.29)-(4.31).

Denote by $\zeta^{h, k}(t, x)$ the second derivative of $\bar{X}(t, x)$ with respect to $x$ towards the directions $h, k \in L^{2}$. Then, $\zeta^{h, k}(t, x)$ satisfies

$$
\begin{aligned}
\frac{d \zeta^{h, k}(t, x)}{d t}= & A \zeta^{h, k}(t, x)+D^{2} \bar{f}(\bar{X}(t, x)) \cdot\left(\eta^{h}(t, x), \eta^{k}(t, x)\right)+D \bar{f}(\bar{X}(t, x)) \cdot \zeta^{h, k}(t, x) \\
& +D_{\xi}\left[\eta^{k}(t, x) \eta^{h}(t, x)\right]+D_{\xi}\left[\bar{X}(t, x) \zeta^{h, k}(t, x)\right]
\end{aligned}
$$

The following lemma gives a control of $\zeta^{h, k}(t, x)$.

Lemma 4.7. Under the conditions $\boldsymbol{A 1}, \boldsymbol{A} 2$ and $\boldsymbol{A 4}$, for any $x \in H^{\theta}$ with $\theta \in(0,1)$, $h, k \in L^{2}, t \in(0, T]$, there exists a constant $C>0$ such that

$$
\left\|\zeta^{h, k}(t, x)\right\| \leqslant C e^{C\|x\|^{5}}\|h\|\|k\| .
$$

Proof. Multiply both sides of the equation (4.32) by $\zeta^{h, k}(t, x)$, integrate with respect to $\xi$, then we obtain

$$
\begin{aligned}
\frac{1}{2} \frac{d}{d t}\left\|\zeta^{h, k}(t, x)\right\|^{2}+\left|\zeta^{h, k}(t, x)\right|_{1}^{2}= & \int_{0}^{1}\left[D^{2} \bar{f}(\bar{X}(t, x)) \cdot\left(\eta^{h}(t, x), \eta^{k}(t, x)\right)\right] \zeta^{h, k}(t, x) d \xi \\
& +\int_{0}^{1}\left[D \bar{f}(\bar{X}(t, x)) \cdot \zeta^{h, k}(t, x)\right] \zeta^{h, k}(t, x) d \xi \\
& +\int_{0}^{1}\left\{D_{\xi}\left[\eta^{k}(t, x) \eta^{h}(t, x)\right]\right\} \zeta^{h, k}(t, x) d \xi \\
& +\int_{0}^{1}\left\{D_{\xi}\left[\bar{X}(t, x) \zeta^{h, k}(t, x)\right]\right\} \zeta^{h, k}(t, x) d \xi \\
= & I_{1}+I_{2}+I_{3}+I_{4} .
\end{aligned}
$$

For $I_{1}$, using (4.16), we get

$$
\left\|I_{1}\right\| \leqslant C e^{C\|x\|^{5}}\|h\|^{2}\|k\|^{2}+\left\|\zeta^{h, k}(t, x)\right\|^{2} .
$$


For $I_{2}$, we have

$$
\left\|I_{2}\right\| \leqslant C\left\|\zeta^{h, k}(t, x)\right\|^{2}
$$

For $I_{3}$, according to Lemma 5.2, we get

$$
\begin{aligned}
\left\|I_{3}\right\| & \leqslant\left|b\left(\eta^{k}(t, x), \eta^{h}(t, x), \zeta^{h, k}(t, x)\right)+b\left(\eta^{h}(t, x), \eta^{k}(t, x), \zeta^{h, k}(t, x)\right)\right| \\
& \leqslant\left(\left\|\eta^{k}(t, x)\right\|\left|\eta^{h}(t, x)\right|_{1}+\left\|\eta^{h}(t, x)\right\|\left|\eta^{k}(t, x)\right|_{1}\right)\left|\zeta^{h, k}(t, x)\right|_{1} \\
& \leqslant C\left(\left\|\eta^{k}(t, x)\right\|\left|\eta^{h}(t, x)\right|_{1}+\left\|\eta^{h}(t, x)\right\|\left|\eta^{k}(t, x)\right|_{1}\right)^{2}+\frac{1}{4}\left|\zeta^{h, k}(t, x)\right|_{1}^{2} .
\end{aligned}
$$

For $I_{4}$, using Lemma 5.2 and the interpolation inequality, we obtain

$$
\begin{aligned}
\left\|I_{4}\right\| & =-\int_{0}^{1}\left[\bar{X}(t, x) \zeta^{h, k}(t, x)\right] D_{\xi} \zeta^{h, k}(t, x) d \xi \\
& \leqslant\left|b\left(\bar{X}(t, x), \zeta^{h, k}(t, x), \zeta^{h, k}(t, x)\right)\right| \\
& \leqslant C\|\bar{X}(t, x)\|\left|\zeta^{h, k}(t, x)\right|_{1}\left|\zeta^{h, k}(t, x)\right|_{\frac{3}{5}} \\
& \leqslant C\|\bar{X}(t, x)\|\left|\zeta^{h, k}(t, x)\right|_{1}^{\frac{8}{5}}\left\|\zeta^{h, k}(t, x)\right\|^{\frac{2}{5}} \\
& \leqslant C\left(1+\|x\|^{5}\right)\left\|\zeta^{h, k}(t, x)\right\|^{2}+\frac{1}{4}\left|\zeta^{h, k}(t, x)\right|_{1}^{2} .
\end{aligned}
$$

Then, combining (4.33)-(4.37), using Lemma 4.4, we get

$$
\begin{aligned}
\left\|\zeta^{h, k}(t, x)\right\|^{2} \leqslant & C e^{C\|x\|^{5}}\|h\|^{2}\|k\|^{2}+C \sup _{0 \leqslant s \leqslant T}\left\|\eta^{k}(s, x)\right\|^{2} \int_{0}^{t}\left|\eta^{h}(s, x)\right|_{1}^{2} d s \\
& +C \sup _{0 \leqslant s \leqslant T}\left\|\eta^{h}(s, x)\right\|^{2} \int_{0}^{t}\left|\eta^{k}(s, x)\right|_{1}^{2} d s+C \int_{0}^{t}\left(1+\|x\|^{5}\right)\left\|\zeta^{h, k}(s, x)\right\|^{2} d s \\
\leqslant & C e^{C\|x\|^{5}}\|h\|^{2}\|k\|^{2}+C \int_{0}^{t}\left(1+\|x\|^{5}\right)\left\|\zeta^{h, k}(s, x)\right\|^{2} d s .
\end{aligned}
$$

The desired result follows by using Gronwall's inequality.

4.2. Properties of $\left(X_{N}^{\varepsilon}, Y_{N}^{\varepsilon}\right)$. This subsection is to establish some properties of $\left(X_{N}^{\varepsilon}, Y_{N}^{\varepsilon}\right)$. For simplicity, we omit the index $N$.

Lemma 4.8. Assume the conditions $\boldsymbol{A} 1, \boldsymbol{A} 2$ and $\boldsymbol{A 4}$ hold.

(1) For any $x \in L^{2}, t \in[0, T]$, there exists a constant $C>0$ such that

$$
\left\|X_{t}^{\varepsilon}\right\| \leqslant C(1+\|x\|) .
$$

(2) For any $x \in H^{\theta}$ with $\theta \in(0,1), y \in L^{2}, \gamma \in\left(1, \frac{3}{2}\right), p \geqslant 1, t \in(0, T]$, there exist $k \in \mathbb{N}$ and a constant $C=C_{p, \theta, \gamma, T}$ such that

$$
\mathbb{E}\left|X_{t}^{\varepsilon}\right|_{\gamma}^{p} \leqslant C t^{-\frac{p(\gamma-\theta)}{2}}\left(1+|x|_{\theta}^{p}+\|y\|^{p}\right) e^{C\|x\|^{k}} .
$$

Proof. Recall that

$$
\frac{d}{d t} X_{t}^{\varepsilon}=A X_{t}^{\varepsilon}+B\left(X_{t}^{\varepsilon}\right)+f\left(X_{t}^{\varepsilon}, Y_{t}^{\varepsilon}\right)
$$

Multiplying both sides of the above equation by $2 X_{t}^{\varepsilon}$ and using (2.7) in A4, we obtain

$$
\frac{1}{2} \frac{d}{d t}\left\|X_{t}^{\varepsilon}\right\|^{2}=-\left|X_{t}^{\varepsilon}\right|_{1}^{2}+\left\langle f\left(X_{t}^{\varepsilon}, Y_{t}^{\varepsilon}\right), X_{t}^{\varepsilon}\right\rangle \leqslant C\left(1+\left\|X_{t}^{\varepsilon}\right\|^{2}\right) .
$$


Then, (4.38) follows by using Gronwall's inequality.

To show (4.39), recall that

$$
X_{t}^{\varepsilon}=e^{t A} x+\int_{0}^{t} e^{(t-s) A} B\left(X_{s}^{\varepsilon}\right) d s+\int_{0}^{t} e^{(t-s) A} f\left(X_{s}^{\varepsilon}, Y_{s}^{\varepsilon}\right) d s:=I_{1}+I_{2}+I_{3} .
$$

For $I_{1},(2.1)$ implies

$$
\left|e^{t A} x\right|_{\gamma} \leqslant C t^{-\frac{\gamma-\theta}{2}}|x|_{\theta}
$$

For $I_{2}$, similarly as the proof of Lemma 4.1, using (4.38), we obtain

$$
\left|\int_{0}^{t} e^{(t-s) A} B\left(X_{s}^{\varepsilon}\right) d s\right|_{\gamma} \leqslant C \int_{0}^{t}(t-s)^{-\frac{1+2 \gamma}{4}}(1+\|x\|)\left|X_{s}^{\varepsilon}\right|_{\gamma} d s .
$$

For the last term, we get

$$
\left|\int_{0}^{t} e^{(t-s) A} f\left(X_{s}^{\varepsilon}, Y_{s}^{\varepsilon}\right) d s\right|_{\gamma} \leqslant C \int_{0}^{t}(t-s)^{-\frac{\gamma}{2}}\left(1+\left\|X_{s}^{\varepsilon}\right\|+\left\|Y_{s}^{\varepsilon}\right\|\right) d s .
$$

Combining (4.40)-(4.43), it follows from the Minkowski inequality that for any $p>1$,

$$
\left[\mathbb{E}\left|X_{t}^{\varepsilon}\right|_{\gamma}^{p}\right]^{1 / p} \leqslant C t^{-\frac{\gamma-\theta}{2}}|x|_{\theta}+C \int_{0}^{t}(t-s)^{-\frac{1+2 \gamma}{4}}(1+\|x\|)\left[\mathbb{E}\left|X_{s}^{\varepsilon}\right|_{\gamma}^{p}\right]^{1 / p} d s+C(1+\|x\|+\|y\|) .
$$

Using Lemma 5.5, we get (4.39).

Similarly to (4.9), for any $x \in H^{\theta}$ with $\delta \in\left(0, \frac{1}{2}\right), y \in L^{2}, \gamma \in(0,1], p \geqslant 1, t \in(0, T]$, there exist $k \in \mathbb{N}$ and a constant $C=C_{p, \delta, \theta, T}$ such that

$$
\mathbb{E}\left|X_{t}^{\varepsilon}\right|_{\gamma}^{p} \leqslant C t^{-\frac{p \gamma(1+\delta-\theta)}{2(1+\delta)}}\left(1+|x|_{\theta}^{p}+\|y\|^{p}\right) e^{C\|x\|^{k}} .
$$

Lemma 4.9. Assume the conditions A1, A2 and A4 hold.

(1) For any $x \in H^{\theta}$ with $\theta \in(0,1), y \in L^{2}, \alpha \in\left(0, \frac{1}{4}\right), 0<s<t \leqslant T$, there exist $k \in \mathbb{N}$ and a constant $C=C_{\theta, \alpha, T}$ such that

$$
\left[\mathbb{E}\left|X_{t}^{\varepsilon}-X_{s}^{\varepsilon}\right|_{1}^{4}\right]^{1 / 4} \leqslant C(t-s)^{\frac{\alpha}{2}} s^{-\frac{1+\alpha-\theta}{2}}\left(|x|_{\theta}+\|y\|+1\right) e^{C\|x\|^{k}} .
$$

(2) For any $x, y \in L^{2}, \alpha \in\left(0, \frac{1}{4}\right), 0<s<t \leqslant T$, there exists a constant $C>0$ such that

$$
\mathbb{E}\left\|Y_{t}^{\varepsilon}-Y_{s}^{\varepsilon}\right\|^{2} \leqslant C\left(\|x\|^{2}+\|y\|^{2}+1\right)\left(s^{-2 \alpha}+\varepsilon^{-2 \alpha}\right)(t-s)^{2 \alpha} .
$$

Proof. The proof of (1) can be carried out in the same way as in Lemma 4.2. For the proof of (2), since the approach here is almost the same as the one in [3], we refer to [3, Proposition A.4] for details.

Lemma 4.10. Under the conditions $\boldsymbol{A 1}, \boldsymbol{A} \mathscr{2}$ and $\boldsymbol{A} 4$, for any $x \in H^{\theta}$ with $\theta \in(0,1)$, $\alpha \in\left(0, \frac{1}{4}\right), 0<t \leqslant T$, there exist $k \in \mathbb{N}$ and a constant $C=C_{\theta, \alpha, T}$ such that

$$
\left[\mathbb{E}\left\|A X_{t}^{\varepsilon}\right\|^{2}\right]^{\frac{1}{2}} \leqslant C t^{-1+\frac{\theta}{2}}\left(|x|_{\theta}^{2}+\|y\|^{2}+1\right) e^{C\|x\|^{k}}+C \varepsilon^{-\alpha} .
$$

Proof. For $0 \leqslant s<t$, we write

$$
\begin{aligned}
X_{t}^{\varepsilon}= & e^{t A} x+\int_{0}^{t} e^{(t-s) A} B\left(X_{t}^{\varepsilon}\right) d s+\int_{0}^{t} e^{(t-s) A}\left[B\left(X_{s}^{\varepsilon}\right)-B\left(X_{t}^{\varepsilon}\right)\right] d s \\
& +\int_{0}^{t} e^{(t-s) A} f\left(X_{t}^{\varepsilon}, Y_{t}^{\varepsilon}\right) d s+\int_{0}^{t} e^{(t-s) A}\left[f\left(X_{s}^{\varepsilon}, Y_{s}^{\varepsilon}\right)-f\left(X_{t}^{\varepsilon}, Y_{t}^{\varepsilon}\right)\right] d s \\
= & I_{1}+I_{2}+I_{3}+I_{4}+I_{5} .
\end{aligned}
$$


For $I_{1}$, using (2.1), we have

$$
\left\|A e^{t A} x\right\| \leqslant C t^{-1+\frac{\theta}{2}}|x|_{\theta}^{2} .
$$

For $I_{2}$, taking $p=4, \gamma=1$ and $\delta=1 / 4$ in (4.44), we get

$$
\begin{aligned}
{\left[\mathbb{E}\left\|A I_{2}\right\|^{2}\right]^{1 / 2}=\left[\mathbb{E}\left\|\left(e^{t A}-I\right) B\left(X_{t}^{\varepsilon}\right)\right\|^{2}\right]^{1 / 2} } & \leqslant C\left[\mathbb{E}\left|X_{t}^{\varepsilon}\right|_{1}^{4}\right]^{1 / 2} \\
& \leqslant C t^{-1+\frac{4 \theta}{5}}\left(1+|x|_{\theta}^{2}+\|y\|^{2}\right) e^{C\|x\|^{k}}
\end{aligned}
$$

For $I_{3}$, using Lemma 5.4, we obtain

$$
\left|A I_{3}\right| \leqslant C \int_{0}^{t} \frac{1}{t-s}\left|B\left(X_{s}^{\varepsilon}\right)-B\left(X_{t}^{\varepsilon}\right)\right| d s \leqslant C \int_{0}^{t} \frac{1}{t-s}\left|X_{t}^{\varepsilon}-X_{s}^{\varepsilon}\right|_{1}\left(\left|X_{t}^{\varepsilon}\right|_{1}+\left|X_{s}^{\varepsilon}\right|_{1}\right) d s .
$$

According to the Minkowski inequality, by Lemmas 4.8 and 4.9, it follows that

$$
\begin{aligned}
{\left[\mathbb{E}\left\|A I_{3}\right\|^{2}\right]^{1 / 2} } & \leqslant C \mathbb{E} \int_{0}^{t} \frac{1}{t-s}\left|X_{t}^{\varepsilon}-X_{s}^{\varepsilon}\right|_{1}\left(\left|X_{t}^{\varepsilon}\right|_{1}+\left|X_{s}^{\varepsilon}\right|_{1}\right) d s \\
& \leqslant C \int_{0}^{t} \frac{1}{t-s}\left\{\mathbb{E}\left[\left|X_{t}^{\varepsilon}-X_{s}^{\varepsilon}\right|_{1}\left(\left|X_{t}^{\varepsilon}\right|_{1}+\left|X_{s}^{\varepsilon}\right|_{1}\right)\right]^{2}\right\}^{1 / 2} d s \\
& \leqslant C \int_{0}^{t} \frac{1}{t-s}\left[\mathbb{E}\left(\left|X_{t}^{\varepsilon}-X_{s}^{\varepsilon}\right|_{1}^{4}\right) \cdot\left(\mathbb{E}\left|X_{t}^{\varepsilon}\right|_{1}^{4}+\mathbb{E}\left|X_{s}^{\varepsilon}\right|_{1}^{4}\right)\right]^{\frac{1}{4}} d s \\
& \leqslant C t^{-1+\frac{9 \theta}{10}}\left(|x|_{\theta}^{2}+\|y\|^{2}+1\right) e^{C\|x\|^{k}} .
\end{aligned}
$$

For $I_{4}$, we have

$$
\mathbb{E}\left\|A I_{4}\right\|=\mathbb{E}\left\|\left(e^{t A}-I\right) f\left(X_{t}^{\varepsilon}, Y_{t}^{\varepsilon}\right)\right\| \leqslant C\left(1+\mathbb{E}\left\|X_{t}^{\varepsilon}\right\|+\mathbb{E}\left\|Y_{t}^{\varepsilon}\right\|\right) \leqslant C(1+\|x\|+\|y\|) .
$$

For $I_{5}$, using the Minkowski inequality and Lemma 4.9, we obtain

$$
\begin{aligned}
{\left[\mathbb{E}\left\|A I_{5}\right\|^{2}\right]^{1 / 2} } & \leqslant C \int_{0}^{t} \frac{1}{t-s}\left[\left(\mathbb{E}\left\|X_{t}^{\varepsilon}-X_{s}^{\varepsilon}\right\|^{2}\right)^{\frac{1}{2}}+\left(\mathbb{E}\left\|Y_{t}^{\varepsilon}-Y_{s}^{\varepsilon}\right\|^{2}\right)^{\frac{1}{2}}\right] d s \\
& \leqslant C t^{-\frac{1}{2}+\frac{\theta}{2}}\left(|x|_{\theta}+\|y\|+1\right) e^{C\|x\|^{k}}+C \varepsilon^{-\alpha} .
\end{aligned}
$$

Combining (4.45)-(4.49) yields the desired result.

Lemma 4.11. Under the conditions $\boldsymbol{A} 1, \boldsymbol{A} 2$ and $\boldsymbol{A} 4$, for any $x \in H^{\theta}$ with $\theta \in(0,1)$, $\alpha \in\left(0, \frac{1}{4}\right), 0<t \leqslant T$, there exist $k \in \mathbb{N}$ and a constant $C=C_{\theta, \alpha, T}$ such that

$$
\mathbb{E}\left\|\frac{d}{d t} X_{t}^{\varepsilon}\right\| \leqslant C t^{-1+\frac{\theta}{2}}\left(|x|_{\theta}^{2}+\|y\|^{2}+1\right) e^{C\|x\|^{k}}+C \varepsilon^{-\alpha} .
$$

Proof. Recall that

$$
\frac{d}{d t} X_{t}^{\varepsilon}=A X_{t}^{\varepsilon}+B\left(X_{t}^{\varepsilon}\right)+f\left(X_{t}^{\varepsilon}, Y_{t}^{\varepsilon}\right)
$$

Choosing $p=2, \gamma=1$ and $\delta=\frac{1}{4}$ in $(4.44)$, we get $\mathbb{E}\left\|B\left(X_{t}^{\varepsilon}\right)\right\| \leqslant C \mathbb{E}\left|X_{t}^{\varepsilon}\right|_{1}^{2} \leqslant C t^{-1+\frac{4 \theta}{5}}\left(|x|_{\theta}^{2}+\right.$ $\left.\|y\|^{2}+1\right)$. It is clear that $\mathbb{E}\left\|f\left(X_{t}^{\varepsilon}, Y_{t}^{\varepsilon}\right)\right\| \leqslant C(1+\|x\|+\|y\|)$. By Lemma 4.10, we have

$$
\mathbb{E}\left\|A X_{t}^{\varepsilon}\right\| \leqslant\left[\mathbb{E}\left\|A X_{t}^{\varepsilon}\right\|^{2}\right]^{1 / 2} \leqslant C t^{-1+\frac{\theta}{2}}\left(|x|_{\theta}^{2}+\|y\|^{2}+1\right) e^{C\|x\|^{k}}+C \varepsilon^{-\alpha} .
$$

The proof is complete. 
4.3. The asymptotic expansion of $\mathbb{E}\left[\phi\left(X_{N}^{\varepsilon}(t)\right)\right]$. For any $x, y \in L^{2}$ and $t \geqslant 0$, set

$$
u_{N}^{\varepsilon}(t, x, y)=\mathbb{E}\left[\phi\left(X_{N}^{\varepsilon}(t, x, y)\right)\right]
$$

and

$$
\bar{u}_{N}(t, x)=\phi\left(\bar{X}_{N}(t, x)\right)
$$

In this subsection, we shall find an asymptotic expansion of $u_{N}^{\varepsilon}$ with respect to $\varepsilon$ :

$$
u_{N}^{\varepsilon}=u_{0}^{N}+\varepsilon u_{1}^{N}+v_{N}^{\varepsilon},
$$

where $v_{N}^{\varepsilon}$ is a residual term, $u_{0}^{N}$ and $u_{1}^{N}$ will be constructed below.

For any $\psi(x, y): L^{2} \times L^{2} \rightarrow \mathbb{R}$ with $\psi \in C^{2}$, we introduce the differential operators:

$$
\begin{gathered}
L_{1}^{N} \psi(x, y)=\left\langle A_{N} x+B_{N}(x)+f_{N}(x, y), D_{x} \psi(x, y)\right\rangle, \\
L_{2}^{N} \psi(x, y)=\left\langle A_{N} x+g_{N}(x, y), D_{y} \psi(x, y)\right\rangle+\frac{1}{2} \operatorname{Tr}\left(D_{y y}^{2} \psi(x, y)\right) .
\end{gathered}
$$

For any $\psi: L^{2} \rightarrow \mathbb{R}$ with $\psi \in C^{1}$, denote by

$$
\bar{L}^{N} \psi(x)=\left\langle A_{N} x+B_{N}(x)+\bar{f}_{N}(x), D_{x} \psi(x)\right\rangle .
$$

Set

$$
L_{N}^{\varepsilon}=L_{1}^{N}+\frac{1}{\varepsilon} L_{2}^{N}
$$

For simplicity, we omit the index $N$. Notice that $\bar{u}$ does not depend on $y$. It is well known that $u^{\varepsilon}$ and $\bar{u}$ satisfy the following Kolmogorov equations:

$$
\left\{\begin{array}{l}
\frac{\partial u^{\varepsilon}(t, x, y)}{\partial t}=L^{\varepsilon} u^{\varepsilon}(t, x, y) \\
u^{\varepsilon}(0, x, y)=\phi(x)
\end{array}\right.
$$

and

$$
\left\{\begin{array}{l}
\frac{\partial \bar{u}(t, x)}{\partial t}=\bar{L} \bar{u}(t, x) \\
\bar{u}(0, x)=\phi(x)
\end{array}\right.
$$

respectively. Using (4.52)-(4.54), we have

$$
\frac{\partial u_{0}}{\partial t}+\varepsilon \frac{\partial u_{1}}{\partial t}+\frac{\partial v^{\varepsilon}}{\partial t}=L_{1} u_{0}+\frac{1}{\varepsilon} L_{2} u_{0}+\varepsilon L_{1} u_{1}+L_{2} u_{1}+L_{1} v^{\varepsilon}+\frac{1}{\varepsilon} L_{2} v^{\varepsilon},
$$

which implies

$$
\left\{\begin{array}{l}
L_{2} u_{0}=0 \\
\frac{\partial u_{0}}{\partial t}=L_{1} u_{0}+L_{2} u_{1} \\
\frac{\partial v^{\varepsilon}}{\partial t}=L^{\varepsilon} v^{\varepsilon}+\varepsilon\left(L_{1} u_{1}-\frac{\partial u_{1}}{\partial t}\right) .
\end{array}\right.
$$

To obtain $u_{0}$ and $u_{1}$, we need the following lemma which is similar to [3, Lemma 4.3]. However, instead of imposing the condition that $f$ is bounded, the coefficient $f$ in our paper is Lipschitz.

Lemma 4.12. Assume the conditions A1, A2 and A4 hold. Fix $x \in L^{2}$.

(1) If $\Psi$ is a Lipschitz continuous function and $\Phi$ is a function of class $C^{2}$ satisfying $L_{2} \Phi=$ $-\Psi$, then for any $y \in L^{2}$, we have

$$
\Phi(y)=\int_{L^{2}} \Phi(z) \mu^{x}(d z)+\int_{0}^{+\infty} \mathbb{E}\left[\Psi\left(Y_{s}^{x, y}\right)\right] d s .
$$


(2) Suppose that $\Psi$ is a Lipschitz continuous function of class $C^{2}$ such that $\int_{L^{2}} \Psi(y) \mu^{x}(d y)=$ 0 . Let $\Phi(y)=\int_{0}^{+\infty} \mathbb{E}\left[\Psi\left(Y_{s}^{x, y}\right)\right] d s$. Then, $\Phi$ is of class $C^{2}$ and satisfies $L_{2} \Phi=-\Psi$. Moreover, there exists a constant $C$ which is independent of $N$ such that for any $y \in L^{2}$

$$
|\Phi(y)| \leqslant C(1+\|x\|+\|y\|)|\Psi|_{\text {Lip }} .
$$

Proof. Since the proof of (1) and the proof of the first part of (2) are similar to [3, Lemma 4.3 , we omit the details. Here we only give a proof of (4.57).

For any $y \in L^{2}$, Proposition 3.9 implies

$$
\left|\mathbb{E}\left[\Psi\left(Y_{s}^{x, y}\right)\right]-\int_{L^{2}} \Psi(z) \mu^{x}(d z)\right| \leqslant C(1+\|x\|+\|y\|) e^{-\frac{\left(\lambda_{1}-L_{g}\right) s}{2}}|\Psi|_{\text {Lip }} .
$$

Noting that $\int_{L^{2}} \Psi(y) \mu^{x}(d y)=0$, we obtain (4.57) by integrating (4.58) with respect to $s$. The proof is complete.

It follows from (4.56) that the function $u_{0}$ is independent of $y$, thus we can write $u_{0}(t, x, y)=$ $u_{0}(t, x)$. We also choose the initial condition $u_{0}(0, x)=\phi(x)$. In view of the second equation in (4.56) and noting that $\int_{L^{2}} L_{2} u_{1}(t, x, y) \mu^{x}(d y)=0$, we have

$$
\begin{aligned}
\frac{\partial u_{0}}{\partial t}(t, x) & =\int_{L^{2}} \frac{\partial u_{0}}{\partial t}(t, x) \mu^{x}(d y) \\
& =\int_{L^{2}} L_{1} u_{0}(t, x) \mu^{x}(d y)+\int_{L^{2}} L_{2} u_{1}(t, x, y) \mu^{x}(d y) \\
& =\left\langle A x+B(x)+\int_{L^{2}} f(x, y) \mu^{x}(d y), D_{x} u_{0}(t, x)\right\rangle \\
& =\bar{L} u_{0}(t, x) .
\end{aligned}
$$

This, together with the uniqueness of the solution to (4.55), implies that $u_{0}=\bar{u}$.

From $\bar{L} u_{0}=L_{1} u_{0}+L_{2} u_{1}$ and the definitions of $\bar{L}$ and $L_{1}$, we deduce that

$$
L_{2} u_{1}(t, x, y)=\left\langle\bar{f}(x)-f(x, y), D_{x} u_{0}(t, x)\right\rangle=:-\chi(t, x, y),
$$

where $\chi$ is of class $C_{b}^{2}$ with respect to $y$, and satisfies that for any $t \geqslant 0$ and $x \in L^{2}$, $\int_{L^{2}} \chi(t, x, y) \mu^{x}(d y)=0$.

According to Lemma 4.12, we obtain

$$
u_{1}(t, x, y)=\int_{0}^{+\infty} \mathbb{E}\left[\chi\left(t, x, Y_{s}^{x, y}\right)\right] d s .
$$

In what follows, we are going to show the regularity of $u_{1}$ with respect to $t$ and $x, y$. In order to avoid the non-integrability at $t=0$, we introduce a parameter $\rho(\varepsilon)=\varepsilon^{\frac{1}{a}}, 0<a \leqslant \frac{\theta}{2}$. By the third equation of (4.56) and Itô's formula, we have

$$
\begin{aligned}
v^{\varepsilon}(t, x, y)= & \mathbb{E}\left[v^{\varepsilon}\left(\rho(\varepsilon), X^{\varepsilon}(t-\rho(\varepsilon), x, y), Y^{\varepsilon}(t-\rho(\varepsilon), x, y)\right)\right] \\
& +\varepsilon \mathbb{E}\left[\int_{\rho(\varepsilon)}^{t}\left(L_{1} u_{1}-\frac{\partial u_{1}}{\partial s}\right)\left(s, X^{\varepsilon}(t-s, x, y), Y^{\varepsilon}(t-s, x, y)\right) d s\right] .
\end{aligned}
$$

Using the expansion (4.52) and the fact $u_{0}=\bar{u}$, we get

$$
\begin{aligned}
& u^{\varepsilon}(t, x, y)-\bar{u}(t, x, y) \\
= & \varepsilon u_{1}(t, x, y)+\mathbb{E}\left[v^{\varepsilon}\left(\rho(\varepsilon), X^{\varepsilon}(t-\rho(\varepsilon), x, y), Y^{\varepsilon}(t-\rho(\varepsilon), x, y)\right)\right] \\
& +\varepsilon \mathbb{E}\left[\int_{\rho(\varepsilon)}^{t}\left(L_{1} u_{1}-\frac{\partial u_{1}}{\partial s}\right)\left(s, X^{\varepsilon}(t-s, x, y), Y^{\varepsilon}(t-s, x, y)\right) d s\right] .
\end{aligned}
$$


Hence, it remains to control each terms in (4.60), which will be shown in the next subsection.

4.4. Estimates of $u_{1}, v^{\varepsilon}, L_{1} u_{1}$ and $\frac{\partial u_{1}}{\partial t}$. Note that the index $N$ is omitted in the equations (4.1) and (4.2).

Lemma 4.13. Under the conditions $\boldsymbol{A 1}, \boldsymbol{A} 2$ and $\boldsymbol{A 4}$, there exists a constant $C>0$ such that for any $0 \leqslant t \leqslant T, x, y \in L^{2}$, we have

$$
\left|u_{1}(t, x, y)\right| \leqslant C e^{C\|x\|^{5}}(1+\|y\|) .
$$

Proof. By (4.59) and Lemma 4.12, we have

$$
\left|u_{1}(t, x, y)\right| \leqslant C(1+\|x\|+\|y\|)|y \longmapsto \chi(t, x, y)|_{\text {Lip }},
$$

where

$$
\chi(t, x, y)=\left\langle f(x, y)-\bar{f}(x), D_{x} u_{0}(t, x)\right\rangle .
$$

Noting that $f$ is Lipschitz, we get that for any $y_{1}, y_{2} \in L^{2}$,

$$
\left|\chi\left(t, x, y_{1}\right)-\chi\left(t, x, y_{2}\right)\right| \leqslant C\left\|y_{1}-y_{2}\right\|\left\|D_{x} u_{0}(t, x)\right\| \text {. }
$$

To bound $\left\|D_{x} u_{0}(t, x)\right\|$, recalling that $u_{0}=\bar{u}$ and $\bar{u}(t, x)=\phi(\bar{X}(t, x))$, we have

$$
D_{x} u_{0}(t, x) \cdot h=D \phi(\bar{X}(t, x)) \cdot \eta^{h}(t, x),
$$

where $\eta^{h}(t, x)=D_{x} \bar{X}(t, x) \cdot h$. Finally the conclusion follows by using Lemma 4.4.

Lemma 4.14. Under the conditions $\boldsymbol{A 1}, \boldsymbol{A} 2$ and $\boldsymbol{A 4}$, for any $x \in H^{\theta}$ with $\theta \in(0,1]$, $y \in L^{2}, 0 \leqslant t \leqslant T$, there exist $k \in \mathbb{N}$ and a constant $C=C_{\theta, T}$ such that

$$
\left|\frac{\partial u_{1}}{\partial t}(t, x, y)\right| \leqslant C t^{-1}\left(1+|x|_{\theta}^{2}\right) e^{C\|x\|^{k}}(1+\|y\|) .
$$

Proof. By the definition of $u_{1}$, we have

$$
\frac{\partial u_{1}}{\partial t}(t, x, y)=\int_{0}^{+\infty} \mathbb{E}\left[\frac{\partial \chi}{\partial t}\left(t, x, Y_{s}^{x, y}\right)\right] d s,
$$

where

$$
\frac{\partial \chi}{\partial t}(t, x, y)=\left\langle f(x, y)-\bar{f}(x), \frac{\partial}{\partial t} D_{x} u_{0}(t, x)\right\rangle .
$$

Using Lemma 4.12, we get

$$
\left|\frac{\partial u_{1}}{\partial t}(t, x, y)\right| \leqslant C(1+\|x\|+\|y\|)\left|y \longmapsto \frac{\partial \chi}{\partial t}(t, x, y)\right|_{\text {Lip }} .
$$

It follows that for any $y_{1}, y_{2} \in L^{2}$,

$$
\begin{aligned}
\left|\frac{\partial \chi}{\partial t}\left(t, x, y_{1}\right)-\frac{\partial \chi}{\partial t}\left(t, x, y_{2}\right)\right| & \leqslant\left\|f\left(x, y_{1}\right)-f\left(x, y_{2}\right)\right\|\left\|\frac{\partial}{\partial t} D_{x} u_{0}(t, x)\right\| \\
& \leqslant C\left\|y_{1}-y_{2}\right\|\left\|\frac{\partial}{\partial t} D_{x} u_{0}(t, x)\right\| .
\end{aligned}
$$


According to Lemmas 4.3, 4.4 and 4.6, for any $h \in L^{2}$, there exist $k \in \mathbb{N}$ and a constant $C=C_{\theta, T}$ such that

$$
\begin{aligned}
\left|\left\langle\frac{\partial}{\partial t} D_{x} u_{0}(t, x), h\right\rangle\right| & =\left|\frac{\partial}{\partial t}\left[D \phi(\bar{X}(t, x)) \cdot \eta^{h}(t, x)\right]\right| \\
& \leqslant\left|D^{2} \phi(\bar{X}(t, x))\left(\eta^{h}(t, x), \frac{d}{d t} \bar{X}(t, x)\right)\right|+\left|D \phi(\bar{X}(t, x)) \cdot \frac{d}{d t} \eta^{h}(t, x)\right| \\
& \leqslant C t^{-1+\frac{\theta}{2}}\left(1+|x|_{\theta}^{2}\right) e^{C\|x\|^{k}}\|h\|+C t^{-1}\left(1+|x|_{\theta}^{2}\right) e^{C\|x\|^{k}}\|h\|,
\end{aligned}
$$

Hence, we obtain

$$
\left\|\frac{\partial}{\partial t} D_{x} u_{0}(t, x)\right\| \leqslant C t^{-1}\left(1+|x|_{\theta}^{2}\right) e^{C\|x\|^{k}} .
$$

The result follows by combining (4.61)-(4.63).

Lemma 4.15. Under the conditions $\boldsymbol{A 1}, \boldsymbol{A} 2$ and $\boldsymbol{A 4}$, there exists a positive constant $C$ such that for any $0 \leqslant t \leqslant T, x \in H^{2}, y \in L^{2}$,

$$
\left|L_{1} u_{1}(t, x, y)\right| \leqslant C e^{C\|x\|^{5}}(1+\|y\|)\left(1+|x|_{1}^{2}+\|y\|+\|A x\|\right) .
$$

Proof. By the definition of $L_{1}$, we have

$$
L_{1} u_{1}(t, x, y)=\left\langle A x+B(x)+f(x, y), D_{x} u_{1}(t, x, y)\right\rangle .
$$

It is easy to see that

$$
|A x+B(x)+f(x, y)| \leqslant C\left(1+|x|_{1}^{2}+\|y\|+\|A x\|\right) .
$$

Thus it remains to estimate $D_{x} u_{1}(t, x, y)$. Recall that $u_{1}$ satisfies

$$
L_{2} u_{1}(t, x, y)=-\chi(t, x, y) \text {. }
$$

For fixed $r>0, h \in L^{2}$, define $\tilde{u}(t, x, y):=\frac{u_{1}(t, x+r h, y)-u_{1}(t, x, y)}{r}$. It follows that

$$
\begin{aligned}
L_{2} \tilde{u}(t, x, y)= & -\frac{\chi(t, x+r h, y)-\chi(t, x, y)}{r} \\
& -\left\langle\frac{g(x+r h, y)-g(x, y)}{r}, D_{y} u_{1}(t, x+r h, y)\right\rangle \\
:= & -\Gamma(t, x, y, h, r) .
\end{aligned}
$$

According to Lemma 4.12, we obtain

$$
\begin{aligned}
& \frac{u_{1}(t, x+r h, y)-u_{1}(t, x, y)}{r}-\int_{H} \frac{u_{1}(t, x+r h, y)-u_{1}(t, x, y)}{r} \mu^{x}(d y) \\
= & \int_{0}^{+\infty} \mathbb{E}\left[\Gamma\left(t, x, Y_{s}^{x, y}, h, r\right)\right] d s .
\end{aligned}
$$

In the same way as in the argument in [3, Section 5.3], as $r \rightarrow 0$, we deduce that

$$
\left|\lim _{r \rightarrow 0} \int_{L^{2}} \frac{u_{1}(t, x+r h, y)-u_{1}(t, x, y)}{r} \mu^{x}(d y)\right| \leqslant C(1+\|x\|)\|h\|
$$

and

$$
\lim _{r \rightarrow 0} \Gamma(t, x, y, h, r)=\Theta(t, x, y) \cdot h:=D_{x} \chi(t, x, y) \cdot h+\left\langle D_{x} g(x, y) \cdot h, D_{y} u_{1}(t, x, y)\right\rangle .
$$


On one hand, by the definition of $\chi$, we have

$$
D_{x} \chi(t, x, y) \cdot h=\left\langle\left(D_{x} f(x, y)-D_{x} \bar{f}(x)\right) \cdot h, D_{x} u_{0}(t, x)\right\rangle+D_{x x}^{2} u_{0}(t, x) \cdot(h, f(x, y)) .
$$

Using condition A4, Lemmas 4.4 and 4.7, it follows that

$$
\left|\left\langle\left(D_{x} f(x, y)-D_{x} \bar{f}(x)\right) \cdot h, D_{x} u_{0}(t, x)\right\rangle\right| \leqslant C e^{C\|x\|^{5}}\|h\|
$$

and

$$
\left|D_{x x}^{2} u_{0}(t, x) \cdot(h, k)\right| \leqslant C e^{C\|x\|^{5}}\|h\|\|k\| .
$$

Then we obtain

$$
\left|D_{x} \chi(t, x, y) \cdot h\right| \leqslant C e^{C\|x\|^{5}}\|h\|+C e^{C\|x\|^{5}}\|h\| \cdot|f(x, y)| \leqslant C e^{C\|x\|^{5}}(1+\|y\|)\|h\| .
$$

On the other hand, using (2.7) and following the argument in [3, Lemma 4.3], we have

$$
\left|\left\langle D_{x} g(x, y) \cdot h, D_{y} u_{1}(t, x, y)\right\rangle\right| \leqslant C\|h\|\left\|D_{y} u_{1}(t, x, y)\right\| \leqslant C\left(1+\|y\|^{2}\right)\|h\| .
$$

Therefore, putting (4.66) and (4.67) together, we get

$$
|\Theta(t, x, y) \cdot h| \leqslant C e^{C\|x\|^{5}}\left(1+\|y\|^{2}\right)\|h\| .
$$

Notice that for any $t, x, r, h$, by the definition of $\Gamma$, we have $\int_{L^{2}} \Gamma(t, x, y, h, r) \mu^{x}(d y)=0$, which implies $\int_{L^{2}} \Theta(t, x, y) \cdot h \mu^{x}(d y)=0$ by using the dominated convergence theorem. Consequently, we obtain

$$
\begin{aligned}
\left|D_{x} u_{1}(t, x, y) \cdot h\right|= & \lim _{r \rightarrow 0} \int_{L^{2}} \frac{u_{1}(t, x+r h, y)-u_{1}(t, x, y)}{r} \mu^{x}(d y) \\
& +\int_{0}^{\infty} \mathbb{E}\left[\Theta\left(t, x, Y_{s}^{x, y}, h\right)\right] d s .
\end{aligned}
$$

One can verify that Lemma 4.12 can be extended to the case where $\Psi$ has the form of quadratic growth as in (4.68). Applying Lemma 4.12 to $\Theta$, from (4.69), we get

$$
\left|D_{x} u_{1}(t, x, y) \cdot h\right| \leqslant C e^{C\|x\|^{5}}(1+\|y\|)\|h\| .
$$

and therefore

$$
\left|L_{1} u_{1}(t, x, y)\right| \leqslant C e^{C\|x\|^{5}}(1+\|y\|)\left(1+|x|_{1}^{2}+\|y\|+\|A x\|\right) .
$$

The result follows by combining (4.64)-(4.65) and (4.70).

Lemma 4.16. Under the conditions $\boldsymbol{A} 1, \boldsymbol{A} 2$ and $\boldsymbol{A 4}$, for any $x \in H^{\theta}$ with $\theta \in(0,1)$, $y \in L^{2}, \alpha \in\left(0, \frac{1}{4}\right), 0 \leqslant t \leqslant T$, there exist $k \in \mathbb{N}$ and a constant $C=C_{\alpha, \theta, T}$ such that

$$
\left|v^{\varepsilon}(\rho(\varepsilon), x, y)\right| \leqslant C \frac{\rho(\varepsilon)^{\frac{\theta}{2}}}{\theta}\left(1+|x|_{\theta}^{2}+\|y\|^{2}\right) e^{C\|x\|^{k}}+C \varepsilon e^{C\|x\|^{5}}(1+\|y\|)+C \rho(\varepsilon) \varepsilon^{-\alpha}
$$

Proof. Using (4.52) and noting that $u_{0}$ is independent of $y$, we write

$$
\begin{aligned}
v^{\varepsilon}(\rho(\varepsilon), x, y) & =\left[u^{\varepsilon}(\rho(\varepsilon), x, y)-u^{\varepsilon}(0, x, y)\right]-\left[u_{0}(\rho(\varepsilon), x)-u_{0}(0, x)\right]-\varepsilon u_{1}(\rho(\varepsilon), x, y) \\
& :=I_{1}-I_{2}-I_{3} .
\end{aligned}
$$


For $I_{1}$, by Lemma 4.11, we have

$$
\begin{aligned}
\left\|I_{1}\right\| & =\left|\int_{0}^{\rho(\varepsilon)} \frac{d}{d t} u^{\varepsilon}(t, x, y) d t\right|=\left\|\int_{0}^{\rho(\varepsilon)} \frac{d}{d t} \mathbb{E}\left[\phi\left(X^{\varepsilon}(t, x, y)\right)\right] d t\right\| \\
& =\left\|\int_{0}^{\rho(\varepsilon)} \mathbb{E}\left[D \phi\left(X^{\varepsilon}(t, x, y)\right) \cdot \frac{d}{d t} X^{\varepsilon}(t, x, y)\right] d t\right\| \\
& \leqslant C \int_{0}^{\rho(\varepsilon)} \mathbb{E}\left\|\frac{d}{d t} X^{\varepsilon}(t, x, y)\right\| d t \leqslant C \frac{\rho(\varepsilon)^{\frac{\theta}{2}}}{\theta}\left(|x|_{\theta}^{2}+\|y\|^{2}+1\right) e^{C\|x\|^{k}}+C \rho(\varepsilon) \varepsilon^{-\alpha} .
\end{aligned}
$$

For $I_{2}$, recalling that $u_{0}=\bar{u}$, by Lemma 4.3 , we get

$$
\begin{aligned}
\left\|I_{2}\right\| & =\left\|\int_{0}^{\rho(\varepsilon)} \frac{d}{d t} u_{0}(t, x) d t\right\|=\left\|\int_{0}^{\rho(\varepsilon)} \frac{d}{d t} \phi(\bar{X}(t, x)) d t\right\| \\
& =\left\|\int_{0}^{\rho(\varepsilon)} D \phi(\bar{X}(t, x)) \cdot \frac{d}{d t} \bar{X}(t, x) d t\right\| \leqslant C \int_{0}^{\rho(\varepsilon)}\left\|\frac{d}{d t} \bar{X}(t, x)\right\| d t \\
& \leqslant \frac{C \rho(\varepsilon)^{\frac{\theta}{2}}}{\theta}\left(1+|x|_{\theta}^{2}\right) e^{C\|x\|^{k}} .
\end{aligned}
$$

For $I_{3}$, it follows from Lemma 4.13 that

$$
\left\|I_{3}\right\| \leqslant C \varepsilon e^{C\|x\|^{5}}(1+\|y\|) .
$$

Putting together (4.71)-(4.74), the result follows.

4.5. Proof of Theorem 2.2. From (4.50)-(4.51) and (4.60), we get

$$
\begin{gathered}
\mathbb{E} \phi\left(X_{N}^{\varepsilon}(t)\right)-\mathbb{E} \phi\left(\bar{X}_{N}(t)\right)=\varepsilon u_{1}(t, x, y)+\mathbb{E}\left[v^{\varepsilon}\left(\rho(\varepsilon), X^{\varepsilon}(t-\rho(\varepsilon), x, y), Y^{\varepsilon}(t-\rho(\varepsilon), x, y)\right)\right] \\
+\varepsilon \mathbb{E}\left[\int_{\rho(\varepsilon)}^{t}\left(L_{1} u_{1}-\frac{\partial u_{1}}{\partial s}\right)\left(s, X^{\varepsilon}(t-s, x, y), Y^{\varepsilon}(t-s, x, y)\right) d s\right] .
\end{gathered}
$$

Using Lemmas 4.13-4.16 and 4.10, (4.44), (4.8), the Hölder inequality, and the fact that $\rho(\varepsilon)=\varepsilon^{\frac{1}{a}}, 0<a \leqslant \frac{\theta}{2}$, we obtain

$$
\begin{aligned}
& \left|\mathbb{E} \phi\left(X_{N}^{\varepsilon}(t)\right)-\mathbb{E} \phi\left(\bar{X}_{N}(t)\right)\right| \\
\leqslant & C \varepsilon e^{C\|x\|^{5}}(1+\|y\|)+C \frac{\rho(\varepsilon)^{\frac{\theta}{2}}}{\theta} \mathbb{E}\left[\left(1+\left|X^{\varepsilon}(t-\rho(\varepsilon))\right|_{\theta}^{2}+\left\|Y^{\varepsilon}(t-\rho(\varepsilon))\right\|^{2}\right) e^{C\left\|X^{\varepsilon}(t-\rho(\varepsilon))\right\|^{k}}\right] \\
& +C \varepsilon \mathbb{E}\left[e^{C\left\|X^{\varepsilon}(t-\rho(\varepsilon))\right\|^{5}}\left(1+\left\|Y^{\varepsilon}(t-\rho(\varepsilon))\right\|\right)\right]+C \rho(\varepsilon) \varepsilon^{-\alpha} \\
& +C \varepsilon \int_{\rho(\varepsilon)}^{t} \mathbb{E}\left[e^{C\left\|X^{\varepsilon}(t-s)\right\|^{5}}\left(1+\left\|Y^{\varepsilon}(t-s)\right\|\right)\left(1+\left|X^{\varepsilon}(t-s)\right|_{1}^{2}+\left\|Y^{\varepsilon}(t-s)\right\|+\left\|A X^{\varepsilon}(t-s)\right\|\right)\right] d s \\
& +C \varepsilon \int_{\rho(\varepsilon)}^{t} s^{-1} \mathbb{E}\left[\left(\left|X^{\varepsilon}(t-s)\right|_{\theta}^{2}+1\right) e^{C\left\|X^{\varepsilon}(t-s)\right\|^{k}}\left(1+\left\|Y^{\varepsilon}(t-s)\right\|\right)\right] d s \\
\leqslant & C \varepsilon+C t^{-\theta+\frac{\theta^{2}}{1+\delta}} \rho(\varepsilon)^{\frac{\theta}{2}}-C \varepsilon \log \varepsilon \leqslant C \varepsilon t^{-\theta+\frac{\theta^{2}}{1+\delta}}-C \varepsilon \log \varepsilon
\end{aligned}
$$

where the constant $C$ is independent of the dimension $N$. Then, letting $N \rightarrow+\infty$ in (4.75), in view of $(4.3)$, the desired result follows. 
4.6. Proof of Theorem 2.3. Since the proof of Theorem 2.3 is similar to that of Theorem 2.2 , we only sketch the difference here. Benefited from a higher regularity of initial value, following the proof of (4.5) in Lemma 4.1, one can verify that if $\theta \in\left(1, \frac{3}{2}\right)$, then

$$
\sup _{t \in[0, T]}\left|\bar{X}_{t}\right|_{\theta} \leqslant C|x|_{\theta} e^{C\|x\|} .
$$

Similarly to the proof of (4.39) in Lemma 4.8, we show that if $\theta \in\left(1, \frac{3}{2}\right)$, then for any $p \geqslant 1$, we have

$$
\sup _{t \in[0, T]} \mathbb{E}\left|X_{t}^{\varepsilon}\right|_{\theta}^{p} \leqslant C\left(1+|x|_{\theta}^{p}+\|y\|^{p}\right) .
$$

Consequently, based on (4.76) and (4.77), one can improve the corresponding results in Lemmas 4.13-4.16. Then we can obtain Theorem 2.3 in an analogous way as in the proof of Theorem 2.2.

\section{ApPEndix}

The following properties of $b(\cdot, \cdot, \cdot)$ and $B(\cdot, \cdot)$ are well-known (for example see [11]).

Lemma 5.1. For any $x, y \in H_{0}^{1}$, it holds that

$$
b(x, x, y)=-\frac{1}{2} b(x, y, x), \quad b(x, y, y)=0 .
$$

Lemma 5.2. Suppose that $\alpha_{i} \geqslant 0(i=1,2,3)$ satisfies one of the following conditions

(1) $\alpha_{i} \neq \frac{1}{2}(i=1,2,3), \alpha_{1}+\alpha_{2}+\alpha_{3} \geqslant \frac{1}{2}$

(2) $\alpha_{i}=\frac{1}{2}$ for some $i, \alpha_{1}+\alpha_{2}+\alpha_{3}>\frac{1}{2}$,

then $b$ is continuous from $H^{\alpha_{1}} \times H^{\alpha_{2}+1} \times H^{\alpha_{3}}$ to $\mathbb{R}$, i.e.

$$
|b(x, y, z)| \leqslant C|x|_{\alpha_{1}}|y|_{\alpha_{2}+1}|z|_{\alpha_{3}} .
$$

The following inequalities can be derived by the above lemma.

Corollary 5.3. For any $x \in H_{0}^{1}$, we have

(1) $\|B(x)\| \leqslant C|x|_{1}^{2}$.

(2) $|B(x)|_{-1} \leqslant C\|x\| \cdot|x|_{1}$.

Lemma 5.4. For any $x, y \in H_{0}^{1}$, we have

(1) $\|B(x)-B(y)\| \leqslant C|x-y|_{1}\left(|x|_{1}+|y|_{1}\right)$.

$(2)|B(x)-B(y)|_{-1} \leqslant C\|x-y\|\left(|x|_{1}+|y|_{1}\right)$.

Similar as the argument in the proof of [25, Theorem 2.6], we can easily obtain the following Gronwall-type inequality, whose proof is based on iteration and we omit the details.

Lemma 5.5 (Gronwall-type inequality). Let $f(t)$ be a non-negative real-valued integrable function on $[0, T]$. For any given $\alpha, \beta \in(0,1)$, if there exist two positive constants $C_{1}, C_{2}$ such that

$$
f(t) \leqslant C_{1} t^{-\alpha}+C_{2} \int_{0}^{t}(t-s)^{-\beta} f(s) d s, \quad \forall t \in[0, T]
$$

then there exists some $k \in \mathbb{N}$ and a positive constant $C:=C_{\alpha, \beta, T}$ such that

$$
f(t) \leqslant C C_{1} t^{-\alpha} e^{C C_{2}^{k}}, \quad \forall t \in[0, T] .
$$


Acknowledge. This paper is partially supported by Key Laboratory of Random Complex Structures and Data Science, PCSDS, Academy of Mathematics and Systems Science, Chinese Academy of Sciences (No: 2008DP173182), by the Priority Academic Program Development of Jiangsu Higher Education Institutions, by Natural Science Foundation of the Higher Education Institutions of Jiangsu Province (No:16KJB110006), by Key Research Program of Frontier Sciences, CAS (No: QYZDB-SSW-SYS009) and the Fundamental Research Funds for the Central Universities (No. WK 3470000008), by NSFC ( No: 11271356, No: 11371041, No: 11431014, No: 11271169, No: 11601196, No: 11771187, No. 11671372, No. 11721101).

\section{REFERENCES}

[1] Bertram, R., Rubin, J. E., Multi-timescale systems and fast-slow analysis. Math. Biosci. 287 (2017), 105-121.

[2] Bogoliubov, N.N., Mitropolsky, Y.A., Asymptotic methods in the theory of Non-linear Oscillations. Gordon and Breach Science Publishers, New York (1961).

[3] Bréhier, C.E., Strong and weak orders in averaging for SPDEs. Stochastic Process. Appl. 122 (2012) 2553-2593.

[4] Cerrai, S., A Khasminskii type averaging principle for stochastic reaction-diffusion equations. Ann.Appl.Probab. 19 (3) (2009) 899-948.

[5] Cerrai, S., Freidlin.M., Averaging principle for stochastic reaction-diffusion equations. Probab.Theory Related Fields 144 (1-2) (2009) 137-177.

[6] Cerrai S., Freidlin, M., Fast flow asymptotics for stochastic incompressible viscous fluids in $\mathbb{R}^{2}$ and SPDEs on graphs. Probab.Theory Related Fields , (2018) 1-45.

[7] Da Prato, G., Debussche, A., m-Dissipativity of Kolmogorov operators corresponding to Burgers equations with space-time white noise. Potential Anal. 26 (2007) 31-55.

[8] Da Prato, G., Zabczyk, J., Stochastic equations in infinite dimensions. Cambridge University Press, 1992.

[9] Da Prato, G., Zabczyk, J., Ergodicity for Infinite Dimensional Systems, Cambridge University Press, 1996.

[10] Da Prato, G., Debussche, A., Temam, R., Stochastic Burgers Equation. Nonlinear Differential Equations Appl. NoDEA 1 (1994) 389-402.

[11] Dong, Z., Xu, T.G., One-dimensional stochastic Burgers equation driven by Lévy processes. J. Funct. Anal. 243 (2007) 631-678.

[12] E, W.; Engquist, B., Multiscale modeling and computations. Notice of AMS, 50 (2003), 1062-1070.

[13] E, W., Liu, D., Vanden-Eijnden.E.: Analysis of multiscale methods for stochastic differential equations. Comm.Pure Appl.Math 58 (11) (2005) 1544-1585.

[14] E, W., Khanin, K., Mazel, A., Sinai, Ya.G., Invariant measures for Burgers equation with stochastic forcing. Ann. of Math, 151 (2000) 877-900.

[15] Fu, H., Liu, J., Strong convergence in stochastic avergaging principle for two time-scales stochastic partial differential equations. J. Math. Anal. Appl. 384 (1) (2011) 70-86.

[16] Fu, H., Wan, L., Liu, J., Strong convergence in averaging principle for stochastic hyperbolic-parabolic equations with two time-scales. Stochastic Process. Appl. 125 (8) (2015) 3255-3279.

[17] Fu, H., Wan, L., Wang, Y., Liu, J., Strong convergence rate in averaging principle for stochastic FitzHugh-Nagumo system with two time-scales. J. Math. Anal. Appl. 416 (2) (2014) 609-628.

[18] Gonzales-Gargate, Ivan I., Ruffino, Paulo R., An averaging principle for diffusions in foliated spaces. Ann.Probab. 44 (1) (2016) 567-588.

[19] Harvey, E., Kirk, V., Wechselberger, M., Sneyd, J., Multiple timescales, mixed mode oscillations and canards in models of intracellular calcium dynamics, J. Nonlinear Sci. 21 (2011) 639-683.

[20] Hu, B., Sun, X., Xie, Y., The Kolmogorov operator and Fokker-Planck equation associated to a stochastic Burgers equation driven by Lévy noise, Illinois J. Math. 58 (1) (2014) 167-205.

[21] Khasminskii, R.Z., On an averging principle for Itô stochastic differential equations. Kibernetica (4) (1968) 260-279. 
[22] Khasminskii, R.Z., Yin, G., Limit behavior of two-time-scale diffusions revised. J.Differential Equations 212 (1) (2005) 85-113.

[23] Krischer, K., Eiswirth, M., Ertl, G., Oscillatory CO oxidation on Pt(110): modeling of temporal selforganization, J. Chem. Phys. 96 (1992), doi: 10.1063/1.462226 .

[24] Li, X.M., An averaging principle for a completely integrable stochastic Hamiltonian system. Nonlinearity 21 (2008) 803-822.

[25] Liu, Y., Zhao, H.Z., Pathwise stationary solutions of stochastic Burgers equations with $L^{2}[0,1]$-noise and stochastic Burgers' integral equations on infinite horizon. arXiv preprint, math/0609344, 2006.

[26] Liu, Y., Zhao, H.Z., Representation of pathwise stationary solutions of stochastic Burgers' equations. Stoch. Dyn., 9(04) (2009) 613-634.

[27] Mastny, Ethan A., Haseltine, Eric L., Rawlings, James B., Two classes of quasi-steady-state model reductions for stochastic kinetics. J.Chem.Phys 127 (2007) 094106.

[28] Mattingly, J. C., Ergodicity of 2D Navier-Stokes Equations with Random Forcing and Large Viscosity. Comm. Math. Phys., 1999, 206(2): 273-288.

[29] Mikikian, M., Cavarroc, M., Couedel, L., Tessier, Y., Boufendi, L., Mixed-mode oscillations in complexplasma instabilities, Phys. Rev. Lett. 100 (2008), doi: 10. 1103/PhysRevLett.100.225005 .

[30] Truman, A., Zhao, H. Z., On stochastic diffusion equations and stochastic Burgers equations. J. Math. Phys., 37(1) (1996) 283-307.

[31] Truman, A., Zhao, H. Z., Stochastic Burgers' equations and their semi-classical expansions. Comm. Math. Phys., 194(1) (1998) 231-248.

[32] Truman, A., Wu, J.L., Stochastic Burgers equation with Lévy space-time white noise, in: Probabilistic Methods in Fluids, Proceedings of the Swansea 2002 Workshop, World Sci. Publishing, River Edge, NJ, (2003) 298-323.

[33] Veretennikov, A.Y., On large deviations in averaging principle for SDEs with full dependence. Ann.Probab. 27 (1999) 284-296.

[34] Wang, W., Roberts, A.J., Average and deviation for slow-fast stochastic partial differential equations. J.Differential Equations 253(5) (2012) 1265-1286.

[35] Wang, W., Roberts, A.J., Duan, J., Large deviations and approximations for slow-fast stochastic reaction-diffusion equations. J.Differential Equations 253(12) (2012) 3501-3522.

[36] Wu, F., Tian, T., Rawlings, J. B., Yin,G., Approximate method for stochastic chemical kinetics with two-time scales by chemical Langevin equations. J. Chem. Phys, 2016, 144(17): 174112.

E-mail address: dzhao@amt.ac.cn

E-mail address: xbsun@jsnu.edu.cn

E-mail address: hui.xiao@univ-ubs.fr

E-mail address: zhaijl@ustc.edu.cn 